\title{
Synthesis, Type II diabetes inhibitory activity, antimicrobial evaluation and docking studies of indeno[1,2-c]pyrazol-4(1H)-ones
}

\author{
Satbir Mor $^{1} \cdot$ Suchita Sindhu ${ }^{1}$ \\ Received: 5 July 2019 / Accepted: 5 October 2019 / Published online: 26 October 2019 \\ (c) Springer Science+Business Media, LLC, part of Springer Nature 2019
}

\begin{abstract}
We report a convenient and efficient synthesis of indeno[1,2-c]pyrazol-4(1H)-ones (4a-o) by the reaction of a variety of 2-acyl-(1H)-indene-1,3(2H)-diones $(\mathbf{1})$ and 2-hydrazinylbenzo[ $d]$ thiazole/2-hydrazinyl-6-substitutedbenzo[ $d]$ thiazoles $(\mathbf{2})$ in the presence of glacial acetic acid in good yields. The structure of the compounds thus prepared were confirmed by analytical and spectral (FT-IR, ${ }^{1} \mathrm{H}$ NMR, ${ }^{13} \mathrm{C}$ NMR, and HRMS) techniques. All the synthesized indeno[1,2-c]pyrazol-4(1H)-ones (4a-o) were assayed for their in vitro Type II diabetes inhibitory activity by using Acarbose as standard drug and in vitro antimicrobial activity utilizing Streptomycin and Fluconazole as reference drugs. Among the synthesized derivatives, $4 \mathbf{e}\left(\mathrm{IC}_{50}=6.71 \mu \mathrm{g} / \mathrm{mL}\right)$ was found to be more potent against $\alpha$-glucosidase enzyme as compared with the standard Acarbose $\left(\mathrm{IC}_{50}=9.35 \mu \mathrm{g} / \mathrm{mL}\right)$ and $\mathbf{4 i}\left(\mathrm{IC}_{50}=11.90 \mu \mathrm{g} / \mathrm{mL}\right)$ exhibited good inhibitory activity against $\alpha$-amylase enzyme as compared with the standard Acarbose $\left(\mathrm{IC}_{50}=22.87 \mu \mathrm{g} / \mathrm{mL}\right)$. Also, all the titled compounds showed good antimicrobial activity. In addition, in vitro $\alpha$-glucosidase and $\alpha$-amylase inhibition were supported by docking studies performed on the derivatives 4e and 4o, respectively.
\end{abstract}

\section{Graphical Abstract}

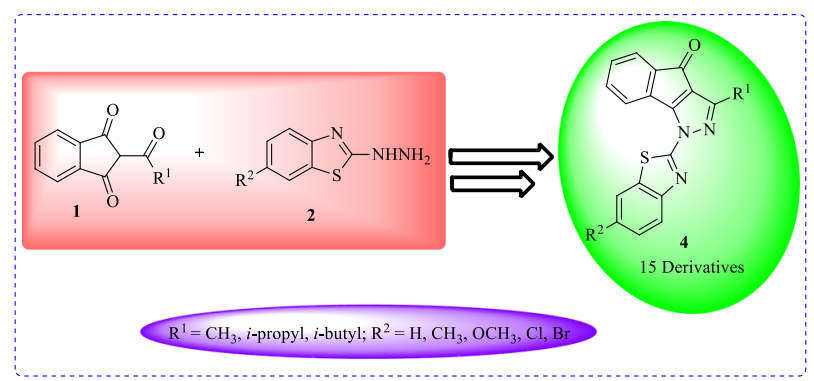

Keywords Indeno[1,2-c]pyrazol-4(1H)-ones $\cdot \alpha$-Glucosidase $\cdot \alpha$-Amylase $\cdot$ Antibacterial $\cdot$ Antifungal $\cdot$ Molecular docking

Supplementary information The online version of this article (https:// doi.org/10.1007/s00044-019-02457-8) contains supplementary material, which is available to authorized users.

Satbir Mor

satbir_mor@yahoo.co.in

1 Department of Chemistry, Guru Jambheshwar University of Science and Technology, Hisar, Haryana 125001, India

\section{Introduction}

Diabetes mellitus is a metabolic disorder resulting from inadequate secretion of insulin characterized by chronic hyperglycemia caused by high calorie diets rich in fat, carbohydrates and proteins (Kumar et al. 2017). The International Diabetes Foundation (IDF) reports that there were 425 million diagnosed cases of diabetes globally in 2017 which is estimated to increase to 629 million by 2045 . Recently, there are more than 46 million diabetics in North America and the Caribbean, 58 million in Europe, 
26 million in South and Central America, 39 million in Middle East and North Africa, 16 million in Africa and 82 million in South-East Asia. There are 352 million people at the risk of developing Type II diabetes (IDF Diabetes Atlas 2017). The emerging factors that contribute to the spread of Type II diabetes, comprising a progressively technological society, food habits with high calorie diets rich in fats and carbohydrates, and an increasingly inactive lifestyle (Wagman et al. 2017). Type II diabetes is associated with hypertension, dyslipidemia, obesity, cardiovascular disease, etc. It may also eventually cause tissue or vascular damage leading to severe diabetic complications such as retinopathy, neuropathy, and nephropathy (Keri et al. 2015). Out of several enzymes known, $\alpha$-amylase and $\alpha$-glucosidase are the key enzymes in the lowering of postprandial hyperglycemia observed in case of Type II diabetes mellitus (T2DM) (Patil et al. 2013). $\alpha$-Amylase inhibits dietary starch from being absorbed into the body system and leads to lowering of blood glucose by the inhibition of salivary and pancreatic amylase (Ajiboye et al. 2016). $\alpha$-Glucosidase inhibitors have been reported to reduce postprandial hyperglycemia in diabetic mellitus resulting in the lowering of glucose absorption by carbohydrate digestion and increases digestion time (Chaudhry et al. 2017).

Likewise, the emergence of bacterial resistance of pathogenic microorganisms is rapidly becoming a major worldwide problem (Mor et al. 2017). Therefore, the demand for new antimicrobial agents is necessary, but now days, it leads to a challenging task for chemists to synthesize new molecules with excellent activity (Kim et al. 2012).

In the recent years, indeno-fused heterocycles are recognized as important frameworks with a broad spectrum of pharmacological properties. Among them, indenopyrazoles have gained substantial attention due to their wide range of biological activities such as antitubercular (Ahsan et al. 2011), tyrosine kinase inhibitors (Khan et al. 2019), CNS agents (Lemke et al. 1978), antioxidant activity (Mor et al. 2019), non-steroidal anti-inflammatory drugs (Lemke et al. 1989), anticancer (Mor et al. 2016), antimicrobial (Shareef et al. 2019), anti-HIV and anticonvulsant activities (Ahsan et al. 2012), and cyclin-dependent kinase (CDK) inhibitors (Singh et al. 2006). Moreover, methyl 3-((6-methoxy-1,4dihydroindeno[1,2-c]pyrazol-3-yl)amino)benzoate was the first indenopyrazole that was reported as a Tubulin Polymerization Inhibitor (Minegishi et al. 2015).

Similarly, benzothiazole is a privileged bicyclic ring system associated with numerous pharmacological activities like antitumor (Gabr et al. 2015), anticonvulsant (Amnerkar and Bhusari 2010), antimicrobial (Chugunova et al. 2015; Kamal et al. 2015), antihelmintic (Sarkar et al. 2013), antileishmanial (Keri et al. 2015), antitubercular (Patel et al. 2013), anti-inflammatory (Shafi et al. 2012), antipsychotic
(Yevich et al. 1986), antioxidant (Bhat and Belagali 2016), antidiabetic (Meltzer-Mats et al. 2013; Kamal et al. 2015) activities, etc. Some of the important marketed drugs involving benzothiazole nucleus are riluzole, sibenadet hydrochloride (Viozan), and pramipexole (Scott and Njardarson 2018). Zopolrestat is another significant drug containing benzothiazole core with antidiabetic effects (Carvalho et al. 2006).

To the best of our knowledge as revealed by literature surveys (Khan et al. 2019), none of hetrocycles with indenopyrazole skeleton have been reported to exhibit antidiabetic effects. Therefore, we thought of synthesizing some new benzothiazole tethered indenopyrazoles to see the additive effect of these moieties towards the preliminary examination of in vitro antidiabetic activity (Doddaramappa et al. 2015). In this perspective and in continuation of our interest in the synthesis of heterocycles containing nitrogen (Zhou et al. 2017; Huang and Huang 2019) and sulfur as heteroatoms and their biological activities herein, we report the synthesis, characterization, $\alpha$-amylase and $\alpha$-glucosidase inhibition, antimicrobial evaluation and docking studies of several benzothiazole tethered indeno[1,2-c]pyrazol-4(1H)ones (4a-0).

\section{Materials and methods}

\section{Chemistry}

All reagents were used without any further purification. Melting points were observed using Electrothermal Melting Point apparatus, LABCO Co., India and are not corrected. The FT-IR spectra were recorded in $\mathrm{KBr}$ on IR affinity-1 FTIR (Shimadzu) spectrophotometer, and results are reported in $\mathrm{cm}^{-1}$. ${ }^{1} \mathrm{H}$ NMR and ${ }^{13} \mathrm{C}$ NMR spectra were recorded on Bruker AVANCE III NMR spectrometer operating at 400 and $100 \mathrm{MHz}$, respectively, with $\mathrm{CDCl}_{3}$ as the solvent and tetramethylsilane (TMS) as the internal standard. Chemical shifts $(\delta)$ are reported in parts per million (ppm), and coupling constants $(J)$ are expressed in Hertz (Hz). HRMS were obtained from Waters Synapt G2Si QTOF and SCIEX $5600^{+}$QTOF mass analyser by using the electrospray ionization (ESI) method. The purity of synthesized compounds was checked by precoated TLC plates (SIL G/UV ${ }_{254}$, ALUGRAM) using a mixture of hexane and ethyl acetate as eluent and visualization was achieved via UV light.

\section{General procedure for the synthesis of 2-acyl-(1H)- indene-1,3(2H)-diones (1)}

2-Acyl-(1H)-indene-1,3(2H)-diones (1) needed for the purpose were prepared via Claisen condensation of 
diethylphthlate and appropriate aliphatic ketones in presence of freshly prepared sodium methoxide following the procedure presented in literature (Mor et al. 2016).

\section{General procedure for the synthesis of 2- hydrazinylbenzo[d]thiazole/2-hydrazinyl-6- substitutedbenzo[d]thiazoles (2)}

Benzo $[d]$ thiazol-2-amine/6-substitutedbenzo $[d]$ thiazol-2amines were prepared by the reaction of aniline/4-substituted anilines and sodium thiocyanate in bromine/glacial acetic acid solution under stirring for $16 \mathrm{~h}$. After completion of reaction, the salt of benzo[d]thiazol-2-amine/6-substitutedbenzo $[d]$ thiazol-2-amines thus obtained was filtered through suction and washed with hexane. Thereafter, the salt was dissolved in water upon warming and the product was precipitated by adding dil. $\mathrm{NaOH}$ solution. The solid thus formed was filtered through suction and recrystallized from ethanol to afford the corresponding amines in high yields (Mor et al. 2017). To a solution of hydrazine hydrochloride in ethylene glycol was added the appropriate benzo[ $d]$ thiazol-2-amine/6-substitutedbenzo $[d]$ thiazol-2-amines in portions with continuous stirring and the resulting mixture was heated to reflux on a heating mantle for $2 \mathrm{~h}$. A fine crystalline solid was separated out on cooling which was filtered, washed with water and crystallized from rectified sprit to yield the corresponding 2-hydrazinylbenzo $[d]$ thiazole/2-hydrazinyl-6-substitutedbenzo [d]thiazoles (2) in good yields (Mor et al. 2017).

\section{General procedure for the synthesis of benzothiazolyl hydrazones (3)}

A solution of equimolar quantities of 2-acyl-( $1 H)$-indene-1,3 $(2 H)$-diones $(1,3 \mathrm{mmol})$ and hydrazines $(2,3 \mathrm{mmol})$ in dry methanol $(15 \mathrm{~mL})$ was heated on a water bath for $15 \mathrm{~min}$ in presence of catalytic amount of glacial acetic acid (4-5 drops). Thereafter, reaction mixture was cooled at room temperature. The solid thus separated out was filtered through suction and recrystallized from ethyl acetate-ethanol to give the corresponding benzothiazolyl hydrazones (3a-o) as orange solids (Sawhney and Lemke 1983; Mor et al. 2019).

\section{General procedure for the synthesis of indeno[1,2-c] pyrazol-4-ones (4a-o)}

Benzothiazolyl hydrazones (3) were charged with glacial acetic acid $(30 \mathrm{~mL})$ and heated to reflux on a heating mantle for $7-9 \mathrm{~h}$ till the completion of reaction as indicated by TLC. The reaction mixture was cooled at room temperature and the solid thus obtained was filtered, and recrystallized from chloroform to furnish the target compounds $\mathbf{4 a - 0}$ in good yields. The physical and spectral data of compounds 4a-o are as follows:
1-(Benzo[d]thiazol-2-yl)-3-methylindeno[1,2-c]pyrazol-4 (1H)-one (4a)<smiles>Cc1nn(-c2nc3ccccc3s2)c2c1C(=O)c1ccccc1-2</smiles>

4a

Yellow solid; yield: $62 \%$; mp $238-240{ }^{\circ} \mathrm{C}$; FTIR $(\mathrm{KBr})$ : $\nu_{\max } 798,1093,1381,1498,1543,1598(\mathrm{C}=\mathrm{N}$ stretch $)$, $1707(\mathrm{C}=\mathrm{O}$ stretch), 2850, 2960 (aliphatic $\mathrm{C}-\mathrm{H}$ stretch), 3068 (aromatic C-H stretch) $\mathrm{cm}^{-1}$; ${ }^{1} \mathrm{H}$ NMR $(400 \mathrm{MHz}$, $\left.\mathrm{CDCl}_{3}\right): \delta=2.45\left(3 \mathrm{H}, \mathrm{s}, \mathrm{CH}_{3}\right), 7.35-7.44(2 \mathrm{H}, \mathrm{m}, \mathrm{Ar}-\mathrm{H})$, $7.50-7.55(2 \mathrm{H}, \mathrm{m}, \mathrm{Ar}-\mathrm{H}), 7.60(1 \mathrm{H}, \mathrm{d}, J=7.20 \mathrm{~Hz}, \mathrm{Ar}-\mathrm{H})$, $7.88(1 \mathrm{H}, \mathrm{d}, J=7.96 \mathrm{~Hz}, \mathrm{Ar}-\mathrm{H}), 8.03(1 \mathrm{H}, \mathrm{d}, J=8.12 \mathrm{~Hz}$, $\mathrm{Ar}-\mathrm{H}), \quad 8.54 \quad(1 \mathrm{H}, \quad \mathrm{d}, \quad J=7.40 \mathrm{~Hz}, \quad 8-\mathrm{H}) ;{ }^{13} \mathrm{C} \quad \mathrm{NMR}$ $\left(100 \mathrm{MHz}, \mathrm{CDCl}_{3}\right): \delta=29.68\left(\mathrm{CH}_{3}\right), 121.36,121.69$, $122.83,124.09,124.29,124.54,125.45,126.75,130.69$, $132.58,133.32,133.58,140.10,148.70,150.95,158.73$, 184.34 (C-4) ppm; HRMS: $\mathrm{m} / \mathrm{z} \quad\left(\mathrm{M}^{+}\right)$Cacld. for $\mathrm{C}_{18} \mathrm{H}_{11} \mathrm{~N}_{3} \mathrm{OS}: 317.0623$, found: $318.0680[\mathrm{M}+\mathrm{H}]^{+}$.

3-Methyl-1-(6-methylbenzo[d]thiazol-2-yl)indeno[1,2-c]pyrazol-4(1H)-one (4b):<smiles>Cc1ccc2nc(-n3nc(C)c4c3-c3ccccc3C4=O)sc2c1</smiles>

4b

Yellow solid; yield $67 \%$; mp $215-218^{\circ} \mathrm{C}$; FTIR $(\mathrm{KBr})$ : $\nu_{\max }$ 798, 1087, 1386, 1500, 1550, $1606(\mathrm{C}=\mathrm{N}$ stretch $)$, $1712(\mathrm{C}=\mathrm{O}$ stretch), 2848, 2954 (aliphatic $\mathrm{C}-\mathrm{H}$ stretch), 3075 (aromatic C-H stretch) $\mathrm{cm}^{-1}$; ${ }^{1} \mathrm{H}$ NMR $(400 \mathrm{MHz}$, $\left.\mathrm{CDCl}_{3}\right): \delta=2.44\left(3 \mathrm{H}, \mathrm{s}, \mathrm{CH}_{3}\right), 2.51\left(3 \mathrm{H}, \mathrm{s}, \mathrm{CH}_{3}\right)$, 7.32-7.38 (2H, m, Ar-H), 7.49-7.53 (2H, m, Ar-H), 7.59 $(1 \mathrm{H}, \mathrm{d}, J=7.08 \mathrm{~Hz}, \mathrm{Ar}-\mathrm{H}), 7.66(1 \mathrm{H}, \mathrm{s}, \mathrm{Ar}-\mathrm{H}), 7.90(1 \mathrm{H}, \mathrm{d}$, $J=8.28 \mathrm{~Hz}$, Ar- $\mathrm{H}), 8.53(1 \mathrm{H}, \mathrm{d}, J=7.40 \mathrm{~Hz}, 8-\mathrm{H}) ;{ }^{13} \mathrm{C}$ NMR $\left(100 \mathrm{MHz}, \mathrm{CDCl}_{3}\right): \delta=22.70\left(\mathrm{CH}_{3}\right), 31.94\left(\mathrm{CH}_{3}\right)$, $121.49,122.38,123.97,124.27,124.54,128.26,130.64$, $132.63,133.45,133.57,135.73,140.16,143.67,148.56$, 148.94, 158.58, 184.38 (C-4); HRMS: $\mathrm{m} / \mathrm{z}\left(\mathrm{M}^{+}\right)$Cacld. for $\mathrm{C}_{19} \mathrm{H}_{13} \mathrm{~N}_{3} \mathrm{OS}$ : 331.0779 , found: $332.0839[\mathrm{M}+\mathrm{H}]^{+}$. 
1-(6-Methoxybenzo[d]thiazol-2-yl)-3-methylindeno[1,2-c] pyrazol-4(1H)-one $(4 \mathrm{c})$ :<smiles>COc1ccc2nc(-n3nc(C)c4c3-c3ccccc3C4=O)sc2c1</smiles>

Yellow solid; yield $69 \%$; mp $210-212{ }^{\circ} \mathrm{C}$; FTIR (KBr): $\nu_{\max } 761,1097,1373,1500,1556,1606(\mathrm{C}=\mathrm{N}$ stretch $)$, $1716(\mathrm{C}=\mathrm{O}$ stretch), 2918, 2962 (aliphatic $\mathrm{C}-\mathrm{H}$ stretch), 3086 (aromatic C-H stretch) $\mathrm{cm}^{-1}$; ${ }^{1} \mathrm{H}$ NMR $(400 \mathrm{MHz}$, $\left.\mathrm{CDCl}_{3}\right): \delta=2.44\left(3 \mathrm{H}, \mathrm{s}, \mathrm{CH}_{3}\right), 3.90\left(3 \mathrm{H}, \mathrm{s}, \mathrm{OCH}_{3}\right), 7.11$ $(1 \mathrm{H}, \mathrm{dd}, J=2.52, J=8.92 \mathrm{~Hz}, \operatorname{Ar}-\mathrm{H}), 7.31-7.40(2 \mathrm{H}, \mathrm{m}$, Ar-H), 7.49-7.60 (2H, m, Ar-H), $7.90(1 \mathrm{H}, \mathrm{d}, J=8.88 \mathrm{~Hz}$, Ar-H), $8.49(1 \mathrm{H}, \quad \mathrm{d}, \quad J=7.40 \mathrm{~Hz}, \quad 8-\mathrm{H}) ;{ }^{13} \mathrm{C} \quad \mathrm{NMR}$ $\left(100 \mathrm{MHz}, \mathrm{CDCl}_{3}\right): \delta=29.79\left(\mathrm{CH}_{3}\right), 55.98 \quad\left(\mathrm{OCH}_{3}\right)$, $115.94,121.49,121.64,123.51,124.36,124.54,130.31$, $130.71,132.70,133.64,134.14,134.77,140.27,145.25$, 148.58, 158.06, 184.46 (C-4); HRMS: m/z $\left(\mathrm{M}^{+}\right)$Calcd. for $\mathrm{C}_{19} \mathrm{H}_{13} \mathrm{~N}_{3} \mathrm{O}_{2} \mathrm{~S}: 347.0728$, found: $348.0786[\mathrm{M}+\mathrm{H}]^{+}$.

\section{1-(6-Chlorobenzo[d]thiazol-2-yl)-3-methylindeno[1,2-c]pyr- azol-4(1H)-one (4d):}<smiles>Cc1nn(-c2nc3ccc(Cl)cc3s2)c2c1C(=O)c1ccccc1-2</smiles>

Yellow solid; yield $62 \%$; mp $228-230{ }^{\circ} \mathrm{C}$; FTIR $(\mathrm{KBr})$ : $\nu_{\max }$ 798, 1099, 1371, 1494, 1546, $1598(\mathrm{C}=\mathrm{N}$ stretch $)$, $1712(\mathrm{C}=\mathrm{O}$ stretch), 2920, 2962 (aliphatic $\mathrm{C}-\mathrm{H}$ stretch), 3089 (aromatic C-H stretch) $\mathrm{cm}^{-1} ;{ }^{1} \mathrm{H}$ NMR $(400 \mathrm{MHz}$, $\left.\mathrm{CDCl}_{3}\right): \delta=2.45\left(3 \mathrm{H}, \mathrm{s}, \mathrm{CH}_{3}\right), 7.36-7.57(4 \mathrm{H}, \mathrm{m}, \mathrm{Ar}-\mathrm{H})$, $7.86(1 \mathrm{H}, \mathrm{d}, J=1.84 \mathrm{~Hz}, \mathrm{Ar}-\mathrm{H}), 7.94(1 \mathrm{H}, \mathrm{d}, J=8.48 \mathrm{~Hz}$, Ar-H), $8.48(1 \mathrm{H}, \quad \mathrm{d}, \quad J=7.40 \mathrm{~Hz}, \quad 8-\mathrm{H}) ;{ }^{13} \mathrm{C} \quad \mathrm{NMR}$ $\left(100 \mathrm{MHz}, \mathrm{CDCl}_{3}\right): \delta=25.29\left(\mathrm{CH}_{3}\right), 121.01,121.41$, $121.58,123.59,124.42,127.59,130.48,130.85,131.29$, $132.42,133.64,134.23,134.49,140.01,148.95,149.50$, 184.30 (C-4); HRMS: $\mathrm{m} / \mathrm{z}\left(\mathrm{M}^{+}\right)$Calcd. for $\mathrm{C}_{18} \mathrm{H}_{10} \mathrm{ClN}_{3} \mathrm{OS}$ : 351.0233, found: $352.0291[\mathrm{M}+\mathrm{H}]^{+}$.
1-(6-Bromobenzo[d]thiazol-2-yl)-3-methylindeno[1,2-c]pyrazol-4(1H)-one (4e):<smiles>Cc1nn(-c2nc3ccc(Br)cc3s2)c2c1C(=O)c1ccccc1-2</smiles>

Yellow solid; yield $70 \%$; mp $208-212^{\circ} \mathrm{C}$; FTIR $(\mathrm{KBr})$ : $\nu_{\max } 765,1099,1394,1496,1546,1598(\mathrm{C}=\mathrm{N}$ stretch $), 1710$ (C=O stretch), 2850, 2958 (aliphatic $\mathrm{C}-\mathrm{H}$ stretch), 3089 (aromatic C-H stretch) $\mathrm{cm}^{-1}$; ${ }^{1} \mathrm{H}$ NMR $\left(400 \mathrm{MHz}, \mathrm{CDCl}_{3}\right)$ : $\delta=2.44\left(3 \mathrm{H}, \mathrm{s}, \mathrm{CH}_{3}\right), 7.36-7.44(2 \mathrm{H}, \mathrm{m}, \mathrm{Ar}-\mathrm{H}), 7.49-7.55$ $(2 \mathrm{H}, \mathrm{m}, \mathrm{Ar}-\mathrm{H}), 7.76(1 \mathrm{H}, \mathrm{d}, J=8.64 \mathrm{~Hz}, \mathrm{Ar}-\mathrm{H}), 7.88(1 \mathrm{H}, \mathrm{d}$, $J=8.64 \mathrm{~Hz}$, Ar-H $), 8.47(1 \mathrm{H}, \mathrm{d}, J=7.40 \mathrm{~Hz}, 8-\mathrm{H}) ;{ }^{13} \mathrm{C}$ NMR $\left(100 \mathrm{MHz}, \mathrm{CDCl}_{3}\right): \delta=29.63\left(\mathrm{CH}_{3}\right), 118.31,121.50$, $123.14,123.86,124.22,124.39,129.99,130.22,130.40$, $130.77,132.35,133.54,134.13,141.53,148.88,150.21$, 164.01, 184.72 (C-4); HRMS: $\mathrm{m} / \mathrm{z}\left(\mathrm{M}^{+}\right)$Cacld. for $\mathrm{C}_{18} \mathrm{H}_{10} \mathrm{BrN}_{3} \mathrm{OS}: 394.9728$, found: $395.9782[\mathrm{M}+\mathrm{H}]^{+}$.

\section{1-(Benzo[d]thiazol-2-yl)-3-isopropylindeno[1,2-c]pyrazol-4} (1H)-one (4f):<smiles>CC(C)c1nn(-c2nc3ccccc3s2)c2c1C(=O)c1ccccc1-2</smiles>

Yellow solid; yield $67 \%$; $200-202{ }^{\circ} \mathrm{C}$; FTIR (KBr): $\nu_{\max }$ 756, 1039, 1398, 1502, 1548, $1598(\mathrm{C}=\mathrm{N}$ stretch $), 1701$ (C=O stretch), 2929, 2972 (aliphatic $\mathrm{C}-\mathrm{H}$ stretch), 3057 (aromatic C-H stretch) $\mathrm{cm}^{-1}$; ${ }^{1} \mathrm{H}$ NMR $\left(400 \mathrm{MHz}, \mathrm{CDCl}_{3}\right)$ : $\delta=1.42\left(6 \mathrm{H}, \mathrm{d}, J=6.96 \mathrm{~Hz},-\mathrm{CH}\left(\mathrm{CH}_{3}\right)_{2}, 3.08-3.15(1 \mathrm{H}\right.$, $\left.\mathrm{m},-\mathrm{CH}\left(\mathrm{CH}_{3}\right)_{2}\right), 7.35-7.43(2 \mathrm{H}, \mathrm{m}, \mathrm{Ar}-\mathrm{H}), 7.50-7.55(2 \mathrm{H}$, $\mathrm{m}, \overline{\mathrm{Ar}-\mathrm{H}}), 7.60(1 \mathrm{H}, \mathrm{d}, J=7.24 \mathrm{~Hz}, \mathrm{Ar}-\mathrm{H}), 7.87(1 \mathrm{H}, \mathrm{d}, J=$ $7.52 \mathrm{~Hz}, \mathrm{Ar}-\mathrm{H}), 8.03(1 \mathrm{H}, \mathrm{d}, J=8.12 \mathrm{~Hz}, \mathrm{Ar}-\mathrm{H}), 8.56(1 \mathrm{H}$, d, $J=7.44 \mathrm{~Hz}, 8-\mathrm{H}) ;{ }^{13} \mathrm{C}$ NMR $\left(100 \mathrm{MHz}, \mathrm{CDCl}_{3}\right): \delta=$ $21.19\left(-\mathrm{CH}\left(\mathrm{CH}_{3}\right)_{2}\right), 28.15\left(-\underline{\mathrm{CH}}\left(\mathrm{CH}_{3}\right)_{2}\right), 121.66,122.81$, $122.90,124.25,124.44,125.38,126.70,130.66,132.81$, 133.38, 133.52, 139.20, 140.13, 150.99, 159.36, 159.52, 183.85 (C-4); HRMS: $\mathrm{m} / \mathrm{z}\left(\mathrm{M}^{+}\right)$Calcd. for $\mathrm{C}_{20} \mathrm{H}_{15} \mathrm{~N}_{3} \mathrm{OS}$ : 345.0936, found: $346.1030[\mathrm{M}+\mathrm{H}]^{+}$. 
3-Isopropyl-1-(6-methylbenzo[d]thiazol-2-yl)indeno[1,2-c] pyrazol-4(1H)-one $(4 \mathrm{~g})$ :<smiles>Cc1ccc2nc(-n3nc(C(C)C)c4c3-c3ccccc3C4=O)sc2c1</smiles>

Yellow solid; yield 67\%; mp $170-174{ }^{\circ} \mathrm{C}$; FTIR (KBr): $\nu_{\max } 759,1055,1381,1502,1548,1602(\mathrm{C}=\mathrm{N}$ stretch $), 1708$ (C=O stretch), 2848, 2966 (aliphatic C-H stretch), 3061 (aromatic C-H stretch) $\mathrm{cm}^{-1}$; $\left.{ }^{1} \mathrm{H} \mathrm{NMR} \mathrm{(400} \mathrm{MHz,} \mathrm{CDCl}_{3}\right)$ : $\delta=1.42\left(6 \mathrm{H}, \mathrm{d}, J=6.92 \mathrm{~Hz},-\mathrm{CH}\left(\underline{\mathrm{CH}}_{3}\right)_{2}, 2.51\left(3 \mathrm{H}, \mathrm{s}, \mathrm{CH}_{3}\right)\right.$, 3.08-3.14 (1H, m, - $\left.\underline{\mathrm{CH}}\left(\mathrm{CH}_{3}\right)_{2}\right), 7.32-7.37$ (2H, m, Ar-H), $7.50(1 \mathrm{H}, \mathrm{t}, J=7.64 \mathrm{~Hz}, \mathrm{Ar}-\mathrm{H}), 7.59(1 \mathrm{H}, \mathrm{d}, J=6.96 \mathrm{~Hz}$, Ar-H), 7.65 (1H, s, Ar-H), $7.90(1 \mathrm{H}, \mathrm{d}, J=8.28 \mathrm{~Hz}, \mathrm{Ar}-\mathrm{H})$, $8.53(1 \mathrm{H}, \mathrm{d}, J=7.40 \mathrm{~Hz}, 8-\mathrm{H}) ;{ }^{13} \mathrm{C} \mathrm{NMR}(100 \mathrm{MHz}$, $\left.\mathrm{CDCl}_{3}\right): \delta=21.21\left(-\mathrm{CH}\left(\underline{\mathrm{CH}_{3}}\right)_{2}\right), 28.16\left(-\underline{\mathrm{CH}}\left(\mathrm{CH}_{3}\right)_{2}\right), 29.71$ $\left(\mathrm{CH}_{3}\right), 121.44,122.35,122.77,124.21,124.43,128.19$, $128.98,130.58,132.86,133.48,135.63,140.19,148.98$, 158.62, 159.12, 159.23, 183.84 (C-4); HRMS: m/z (M $\left.{ }^{+}\right)$ Calcd. for $\mathrm{C}_{21} \mathrm{H}_{17} \mathrm{~N}_{3} \mathrm{OS}$ : 359.1092, found: $360.1158[\mathrm{M}+\mathrm{H}]^{+}$.

\section{3-Isopropyl-1-(6-methoxybenzo[d]thiazol-2-yl)indeno[1,2-c] pyrazol-4(1H)-one $(4 \mathrm{~h})$ :}<smiles>COc1ccc2nc(-n3nc(C(C)C)c4c3-c3ccccc3C4=O)sc2c1</smiles>

Yellow solid; yield $65 \%$; mp 212-214 ${ }^{\circ} \mathrm{C}$; FTIR (KBr): $\nu_{\max }$ 759, 1089, 1398, 1504, 1550, 1608 (C=N stretch), $1703(\mathrm{C}=\mathrm{O}$ stretch), 2837, 2962 (aliphatic $\mathrm{C}-\mathrm{H}$ stretch), 3078 (aromatic C-H stretch) $\mathrm{cm}^{-1}$; ${ }^{1} \mathrm{H} \mathrm{NMR}(400 \mathrm{MHz}$, $\left.\mathrm{CDCl}_{3}\right): \quad \delta=1.42 \quad\left(6 \mathrm{H}, \quad \mathrm{d}, \quad J=6.92 \mathrm{~Hz}, \quad-\mathrm{CH}\left(\mathrm{CH}_{3}\right)_{2}\right.$, 3.07-3.14 (1H, m,- $\left.\mathrm{CH}\left(\mathrm{CH}_{3}\right)_{2}\right), 3.90\left(3 \mathrm{H}, \mathrm{s}, \mathrm{OCH}_{3}\right), 7.11$ $(1 \mathrm{H}, \mathrm{dd}, J=2.36, J=8.92 \mathrm{~Hz}, \mathrm{Ar}-\mathrm{H}), 7.32-7.37(2 \mathrm{H}, \mathrm{m}$, Ar-H), $7.50(1 \mathrm{H}, \mathrm{t}, J=7.56 \mathrm{~Hz}, \operatorname{Ar}-\mathrm{H}), 7.59(1 \mathrm{H}, \mathrm{d}, J=$ $7.24 \mathrm{~Hz}, \mathrm{Ar}-\mathrm{H}), 7.90(1 \mathrm{H}, \mathrm{d}, J=8.92 \mathrm{~Hz}, \mathrm{Ar}-\mathrm{H}), 8.50(1 \mathrm{H}$, $\mathrm{d}, J=7.44 \mathrm{~Hz}, 8-\mathrm{H}) ;{ }^{13} \mathrm{C} \mathrm{NMR}\left(100 \mathrm{MHz}, \mathrm{CDCl}_{3}\right): \delta=$ $21.22\left(-\mathrm{CH}\left(\underline{\mathrm{CH}}_{3}\right)_{2}\right), 28.14\left(-\underline{\mathrm{CH}}\left(\mathrm{CH}_{3}\right)_{2}\right), 55.94\left(\mathrm{OCH}_{3}\right)$, $104.51,115.71,122.63,123.38,124.21,124.33,130.56$,
$132.83,133.45,134.72,140.20,145.19,157.21,157.91$, 158.85, 159.17, 183.83 (C-4); HRMS: m/z (M $\left.{ }^{+}\right)$Calcd. for $\mathrm{C}_{21} \mathrm{H}_{17} \mathrm{~N}_{3} \mathrm{O}_{2} \mathrm{~S}$ : 375.1041 , found: $376.1103[\mathrm{M}+\mathrm{H}]^{+}$.

1-(6-Chlorobenzo[d]thiazol-2-yl)-3-isopropylindeno[1,2-c] pyrazol-4(1H)-one (4i):<smiles>CC(C)c1nn(-c2nc3ccc(Cl)cc3s2)c2c1C(=O)c1ccccc1-2</smiles>

Yellow solid; yield 70\%; mp $182-184{ }^{\circ} \mathrm{C}$; FTIR (KBr): $\nu_{\max } 763,1109,1381,1500,1544,1597(\mathrm{C}=\mathrm{N}$ stretch $)$, 1703 ( $\mathrm{C}=\mathrm{O}$ stretch), 2870, 2964 (aliphatic C-H stretch), 3086 (aromatic C-H stretch) $\mathrm{cm}^{-1}$; ${ }^{1} \mathrm{H} \mathrm{NMR}(400 \mathrm{MHz}$, $\left.\mathrm{CDCl}_{3}\right): \quad \delta=1.42 \quad\left(6 \mathrm{H}, \quad \mathrm{d}, \quad J=6.92 \mathrm{~Hz}, \quad-\mathrm{CH}\left(\underline{\mathrm{CH}}_{3}\right)_{2}\right.$, 3.07-3.14 (1H, m, - $\left.\underline{\mathrm{CH}}\left(\mathrm{CH}_{3}\right)_{2}\right), 7.36(1 \mathrm{H}, \mathrm{t}, J=7 . \overline{48} \mathrm{~Hz}$, Ar-H), 7.45-7.51 (2H, m, Ar-H), $7.59(1 \mathrm{H}, \mathrm{d}, J=7.20 \mathrm{~Hz}$, Ar-H), $7.82(1 \mathrm{H}, \mathrm{d}, J=1.64 \mathrm{~Hz}, \mathrm{Ar}-\mathrm{H}), 7.91(1 \mathrm{H}, \mathrm{d}, J=$ $8.68 \mathrm{~Hz}, \mathrm{Ar}-\mathrm{H}), 8.46(1 \mathrm{H}, \mathrm{d}, J=7.40 \mathrm{~Hz}, 8-\mathrm{H}) ;{ }^{13} \mathrm{C} \mathrm{NMR}$ $\left(100 \mathrm{MHz}, \mathrm{CDCl}_{3}\right): \delta=21.13\left(-\mathrm{CH}\left(\underline{\mathrm{CH}}_{3}\right)_{2}\right), 28.12(-\underline{\mathrm{CH}}$ $\left.\left(\mathrm{CH}_{3}\right)_{2}\right), 121.29,123.06,123.49,124.30,127.46,130 . \overline{73}$, $131.17,132.61,133.47,134.53,140.01,149.51,159.30$, 159.48, 159.80, 183.67 (C-4); HRMS: m/z (M $\left.{ }^{+}\right)$Calcd. for $\mathrm{C}_{20} \mathrm{H}_{14} \mathrm{ClN}_{3} \mathrm{OS}$ : 379.0546, found: $380.0605[\mathrm{M}+\mathrm{H}]^{+}$.

1-(6-Bromobenzo[d]thiazol-2-yl)-3-isopropylindeno[1,2-c] pyrazol-4(1H)-one (4j):<smiles>CC(C)c1nn(-c2nc3ccc(Br)cc3s2)c2c1C(=O)c1ccccc1-2</smiles>

Yellow solid; yield 65\%; mp 190-194 ${ }^{\circ} \mathrm{C}$; FTIR (KBr): $\nu_{\max }$ 738, 1078, 1390, 1502, 1539, 1593 ( $\mathrm{C}=\mathrm{N}$ stretch), 1707 (C=O stretch), 2870, 2960 (aliphatic C-H stretch), 3062 (aromatic C-H stretch) $\mathrm{cm}^{-1}$; ${ }^{1} \mathrm{H}$ NMR (400 MHz, $\mathrm{CDCl}_{3}$ ): $\delta=1.41\left(6 \mathrm{H}, \mathrm{d}, J=6.92 \mathrm{~Hz},-\mathrm{CH}\left(\mathrm{CH}_{3}\right)_{2}, 3.07-3.14(1 \mathrm{H}, \mathrm{m},-\right.$ $\left.\underline{\mathrm{CH}}\left(\mathrm{CH}_{3}\right)_{2}\right), 7.37(1 \mathrm{H}, \mathrm{t}, J=7.44 \mathrm{~Hz}, \mathrm{Ar}-\mathrm{H}), 7.51(1 \mathrm{H}, \mathrm{t}, J=$ $7.56 \mathrm{~Hz}, \mathrm{Ar}-\mathrm{H}), 7.59-7.64$ (2H, m, Ar-H), 7.88 (1H, d, J= $8.64 \mathrm{~Hz}, \mathrm{Ar}-\mathrm{H}), 8.00$ (1H, d, $J=1.76 \mathrm{~Hz}, \mathrm{Ar}-\mathrm{H}), 8.48(1 \mathrm{H}, \mathrm{d}$, 
$J=7.28 \mathrm{~Hz}, 8-\mathrm{H}) ;{ }^{13} \mathrm{C}$ NMR $\left(100 \mathrm{MHz}, \mathrm{CDCl}_{3}\right): \delta=21.15$ $\left(-\mathrm{CH}\left(\mathrm{CH}_{3}\right)_{2}\right), 28.14\left(-\mathrm{CH}\left(\mathrm{CH}_{3}\right)_{2}\right), 118.70,123.11,123.90$, $124.23,124.34,130.22,130.79,132.63,133.53,134.99$, $140.02,144.69,149.89,159.39,159.55,159.83,183.74$ (C-4); HRMS: $\mathrm{m} / \mathrm{z}\left(\mathrm{M}^{+}\right)$Calcd. for $\mathrm{C}_{20} \mathrm{H}_{14} \mathrm{BrN}_{3} \mathrm{OS}$ : 423.0041, found: $424.0100[\mathrm{M}+\mathrm{H}]^{+}$.

\section{1-(benzo[d]thiazol-2-yl)-3-isobutylindeno[1,2-c]pyrazol-4} (1H)-one (4k):<smiles>CC(C)Cc1nn(-c2nc3ccccc3s2)c2c1C(=O)c1ccccc1-2</smiles>

Yellow solid; yield 57\%; mp 222-226 ${ }^{\circ} \mathrm{C}$; FTIR (KBr): $\nu_{\max }$ 761, 1091, 1390, 1494, 1556, 1597 (C=N stretch), 1705 (C=O stretch), 2873, 2958 (aliphatic C-H stretch), 3066 (aromatic C-H stretch) $\mathrm{cm}^{-1} ;{ }^{1} \mathrm{H} \mathrm{NMR}\left(400 \mathrm{MHz}, \mathrm{CDCl}_{3}\right): \delta$ $=1.01\left(6 \mathrm{H}, \mathrm{d}, J=6.60 \mathrm{~Hz},-\mathrm{CH}_{2} \mathrm{CH}\left(\underline{\mathrm{CH}}_{3}\right)_{2}\right), 2.26-2.33(1 \mathrm{H}$, $\left.\mathrm{m},-\mathrm{CH}_{2} \underline{\mathrm{CH}}\left(\mathrm{CH}_{3}\right)_{2}\right), 2.66\left(2 \mathrm{H}, \mathrm{d}, J=7.28 \mathrm{~Hz},-\underline{\mathrm{CH}}_{2} \mathrm{CH}\left(\mathrm{CH}_{3}\right)\right.$ 2), 7.34-7.43 (2H, m, Ar-H), 7.50-7.55 (2H, m, Ar-H), 7.59 $(1 \mathrm{H}, \mathrm{d}, J=7.20 \mathrm{~Hz}, \mathrm{Ar}-\mathrm{H}), 7.87(1 \mathrm{H}, \mathrm{d}, J=8.00 \mathrm{~Hz}, \mathrm{Ar}-\mathrm{H})$, $8.03(1 \mathrm{H}, \mathrm{d}, J=8.12 \mathrm{~Hz}, \mathrm{Ar}-\mathrm{H}), 8.55(1 \mathrm{H}, \mathrm{d}, J=7.44 \mathrm{~Hz}, 8-$ $\mathrm{H}) ;{ }^{13} \mathrm{C} \mathrm{NMR}\left(100 \mathrm{MHz}, \mathrm{CDCl}_{3}\right): \delta=22.43\left(-\mathrm{CH}_{2} \mathrm{CH}\left(\underline{\mathrm{CH}}_{3}\right)\right.$ 2), $27.79\left(-\mathrm{CH}_{2} \underline{\mathrm{CH}}\left(\mathrm{CH}_{3}\right)_{2}\right), 36.32\left(-\mathrm{CH}_{2} \mathrm{CH}\left(\mathrm{CH}_{3}\right)_{2}\right), 121.63$, $122.79,123.98,124.24,124.43,125.39,126.69,130.62$, $132.67,133.34,133.53,140.13,148.32,150.93,152.78$, 158.81, 184.14 (C-4); HRMS: $\mathrm{m} / \mathrm{z} \quad\left(\mathrm{M}^{+}\right)$Calcd. for $\mathrm{C}_{21} \mathrm{H}_{17} \mathrm{~N}_{3} \mathrm{OS}$ : 359.1092, found: $360.1155[\mathrm{M}+\mathrm{H}]^{+}$.

\section{3-Isobutyl-1-(6-methylbenzo[d]thiazol-2-yl)indeno[1,2-c] pyrazol-4(1H)-one (4I):}<smiles>Cc1ccc2nc(-n3nc(CC(C)C)c4c3-c3ccccc3C4=O)sc2c1</smiles>

Yellow solid; yield 54\%; mp 186-190 ${ }^{\circ} \mathrm{C}$; FTIR (KBr): $\nu_{\max } 773,1134,1444,1465,1562,1602(\mathrm{C}=\mathrm{N}$ stretch $)$, 1693 (C=O stretch), 2868, 2953 (aliphatic C-H stretch), 3064 (aromatic C-H stretch) $\mathrm{cm}^{-1} ;{ }^{1} \mathrm{H}$ NMR $(400 \mathrm{MHz}$, $\left.\mathrm{CDCl}_{3}\right): \delta=1.00\left(6 \mathrm{H}, \mathrm{d}, J=6.60 \mathrm{~Hz},-\mathrm{CH}_{2} \mathrm{CH}\left(\underline{\mathrm{CH}}_{3}\right)_{2}\right)$, 1.96-2.02 $\left(1 \mathrm{H}, \mathrm{m},-\mathrm{CH}_{2} \underline{\mathrm{CH}}\left(\mathrm{CH}_{3}\right)_{2}\right), 2.41\left(3 \mathrm{H}, \mathrm{s}, \mathrm{CH}_{3}\right)$, $2.78\left(2 \mathrm{H}, \mathrm{d}, J=7.44 \mathrm{~Hz},-\mathrm{CH}_{2} \mathrm{CH}\left(\mathrm{CH}_{3}\right)_{2}\right), 7.31-7.40$ $(2 \mathrm{H}, \mathrm{m}, \mathrm{Ar}-\mathrm{H}), 7.45-7.51(2 \mathrm{H}, \mathrm{m}, \mathrm{Ar}-\mathrm{H}), 7.77(1 \mathrm{H}, \mathrm{t}, J$ $=8.00 \mathrm{~Hz}, \mathrm{Ar}-\mathrm{H}), 8.02(1 \mathrm{H}, \mathrm{d}, J=7.56 \mathrm{~Hz}, \mathrm{Ar}-\mathrm{H}), 8.40$ $(1 \mathrm{H}, \mathrm{d}, J=9.28 \mathrm{~Hz}, 8-\mathrm{H}) ;{ }^{13} \mathrm{C} \mathrm{NMR}\left(100 \mathrm{MHz}, \mathrm{CDCl}_{3}\right)$ : $\delta=21.44 \quad\left(-\mathrm{CH}_{2} \mathrm{CH}\left(\mathrm{CH}_{3}\right)_{2}\right), \quad 22.31 \quad\left(\mathrm{CH}_{3}\right), \quad 28.00$ $\left(-\mathrm{CH}_{2} \mathrm{CH}\left(\mathrm{CH}_{3}\right)_{2}\right), \quad 37.09 \quad\left(-\mathrm{CH}_{2} \mathrm{CH}\left(\mathrm{CH}_{3}\right)_{2}\right), \quad 119.77$, $120.95,121.95,123.88,125.12,127.58,128.98,130.64$, 132.01, 139.18, 140.27, 148.66, 150.06, 151.58, 154.85, 159.83, 182.64 (C-4); HRMS: $\mathrm{m} / \mathrm{z}\left(\mathrm{M}^{+}\right)$Calcd. for $\mathrm{C}_{22} \mathrm{H}_{19} \mathrm{~N}_{3} \mathrm{OS}$ : 373.1249 , found: $374.1309[\mathrm{M}+\mathrm{H}]^{+}$.

3-Isobutyl-1-(6-methoxybenzo[d]thiazol-2-yl)indeno[1,2c]pyrazol-4(1H)-one $(4 \mathrm{~m})$ :<smiles>COc1ccc2nc(-n3nc(CC(C)C)c4c3-c3ccccc3C4=O)sc2c1</smiles>

Yellow solid; yield: 56\%; mp $158-160{ }^{\circ} \mathrm{C}$; FTIR $(\mathrm{KBr}): \nu_{\max } 759,1089,1390,1496,1550,1605(\mathrm{C}=\mathrm{N}$ stretch), $1705(\mathrm{C}=\mathrm{O}$ stretch), 2868, 2956 (aliphatic $\mathrm{C}-\mathrm{H}$ stretch), 3061 (aromatic $\mathrm{C}-\mathrm{H}$ stretch) $\mathrm{cm}^{-1} ;{ }^{1} \mathrm{H}$ NMR $\left(400 \mathrm{MHz}, \quad \mathrm{CDCl}_{3}\right): \quad \delta=1.02 \quad(6 \mathrm{H}, \quad \mathrm{d}, \quad J=6.6 \mathrm{~Hz}$, $\left.-\mathrm{CH}_{2} \mathrm{CH}\left(\underline{\mathrm{CH}}_{3}\right)_{2}\right), \quad 2.26-2.33\left(1 \mathrm{H}, \mathrm{m},-\mathrm{CH}_{2} \underline{\mathrm{CH}}\left(\mathrm{CH}_{3}\right)_{2}\right)$, $2.66\left(2 \mathrm{H}, \mathrm{d}, J=7.28 \mathrm{~Hz},-\mathrm{CH}_{2} \mathrm{CH}\left(\mathrm{CH}_{3}\right)_{2}\right), 3.91(3 \mathrm{H}, \mathrm{s}$, $\left.\mathrm{OCH}_{3}\right), \quad 7.12(1 \mathrm{H}, \quad \mathrm{dd}, \quad J=2.28, \quad 6.60 \mathrm{~Hz}, \quad \mathrm{Ar}-\mathrm{H})$, $7.32-7.37(2 \mathrm{H}, \mathrm{m}, \mathrm{Ar}-\mathrm{H}), 7.51(1 \mathrm{H}, \mathrm{t}, J=7.48 \mathrm{~Hz}, \mathrm{Ar}-\mathrm{H})$, $7.59(1 \mathrm{H}, \mathrm{d}, J=7.24 \mathrm{~Hz}, \mathrm{Ar}-\mathrm{H}), 7.91(1 \mathrm{H}, \mathrm{d}, J=$ $8.92 \mathrm{~Hz}$, Ar-H), $8.50(1 \mathrm{H}, \mathrm{d}, J=7.48 \mathrm{~Hz}, 8-\mathrm{H}) ;{ }^{13} \mathrm{C}$ NMR $\left(100 \mathrm{MHz}, \mathrm{CDCl}_{3}\right): \delta=22.52\left(-\mathrm{CH}_{2} \mathrm{CH}\left(\underline{\mathrm{CH}}_{3}\right)_{2}\right)$, $29.74\left(-\mathrm{CH}_{2} \mathrm{CH}\left(\mathrm{CH}_{3}\right)_{2}\right), 36.41\left(-\mathrm{CH}_{2} \mathrm{CH}\left(\mathrm{CH}_{3}\right)_{2}\right), 55.94$ $\left(\mathrm{OCH}_{3}\right), 104.56,115.82,123.45,123.83,124.28,124.41$, 127.13, 130.61, 132.79, 133.55, 134.79, 136.96, 140.30, 145.23, 150.48, 152.66, 158.00, 184.23 (C-4); HRMS: $\mathrm{m} / \mathrm{z}\left(\mathrm{M}^{+}\right)$Calcd. for $\mathrm{C}_{22} \mathrm{H}_{19} \mathrm{~N}_{3} \mathrm{O}_{2} \mathrm{~S}: 389.1198$, found: $390.1258[\mathrm{M}+\mathrm{H}]^{+}$. 
1-(6-Chlorobenzo[d]thiazol-2-yl)-3-isobutylindeno[1,2-c] pyrazol-4(1H)-one $(4 \mathrm{n})$ :<smiles>CC(C)Cc1nn(-c2nc3ccc(Cl)cc3s2)c2c1C(=O)c1ccccc1-2</smiles>

Yellow solid; yield: 49\%; mp 140-142 ${ }^{\circ} \mathrm{C}$; FTIR (KBr): $\nu_{\max } 775,1109,1388,1494,1537,1593(\mathrm{C}=\mathrm{N}$ stretch $)$, $1708(\mathrm{C}=\mathrm{O}$ stretch), 2870, 2962 (aliphatic $\mathrm{C}-\mathrm{H}$ stretch), 3088 (aromatic C-H stretch) $\mathrm{cm}^{-1}$; ${ }^{1} \mathrm{H} \mathrm{NMR}(400 \mathrm{MHz}$, $\left.\mathrm{CDCl}_{3}\right): \delta=1.02\left(6 \mathrm{H}, \mathrm{d}, J=6.60 \mathrm{~Hz},-\mathrm{CH}_{2} \mathrm{CH}\left(\underline{\mathrm{CH}}_{3}\right)_{2}\right)$, 2.26-2.33 (1H, m, $\left.-\mathrm{CH}_{2} \underline{\mathrm{CH}}\left(\mathrm{CH}_{3}\right)_{2}\right), 2.66(2 \mathrm{H}, \mathrm{d}, J=$ $\left.7.32 \mathrm{~Hz},-\underline{\mathrm{CH}}_{2} \mathrm{CH}\left(\mathrm{CH}_{3}\right)_{2}\right), 7.35-7.45(2 \mathrm{H}, \mathrm{m}, \mathrm{Ar}-\mathrm{H}), 7.70$ (1H, t $J=7.40 \mathrm{~Hz}$, Ar-H), 7.80-7.85 (2H, m, Ar-H), 7.95 $(1 \mathrm{H}, \mathrm{d}, J=8.68 \mathrm{~Hz}, \mathrm{Ar}-\mathrm{H}), 8.49(1 \mathrm{H}, \mathrm{d}, J=7.48 \mathrm{~Hz}, 8-\mathrm{H})$; ${ }^{13} \mathrm{C} \mathrm{NMR}\left(100 \mathrm{MHz}, \mathrm{CDCl}_{3}\right): \delta=22.47\left(-\mathrm{CH}_{2} \mathrm{CH}\left(\underline{\mathrm{CH}}_{3}\right)_{2}\right)$, $27.82\left(-\mathrm{CH}_{2} \underline{\mathrm{CH}}\left(\mathrm{CH}_{3}\right)_{2}\right), 36.34\left(-\underline{\mathrm{CH}}_{2} \mathrm{CH}\left(\mathrm{CH}_{3}\right)_{2}\right), 120.97$, $121.36,121.49,123.57,124.37,124.47,127.28,127.54$, $130.78,133.58,140.09,149.54,151.28,152.08,153.03$, 158.93, 185.16 (C-4); HRMS: $\mathrm{m} / \mathrm{z} \quad\left(\mathrm{M}^{+}\right)$Calcd. for $\mathrm{C}_{21} \mathrm{H}_{16} \mathrm{ClN}_{3} \mathrm{OS}$ : 393.0703, found: $394.0763[\mathrm{M}+\mathrm{H}]^{+}$.

\section{1-(6-Bromobenzo[d]thiazol-2-yl)-3-isobutylindeno[1,2-c] pyrazol-4(1H)-one $(40$}<smiles>CC(C)Cc1nn(-c2nc3ccc(Br)cc3s2)c2c1C(=O)c1ccccc1-2</smiles>

Yellow solid; yield $78 \%$; mp $168-170{ }^{\circ} \mathrm{C}$; FTIR (KBr): $\nu_{\max } 763,1097,1392,1494,1537,1593(\mathrm{C}=\mathrm{N}$ stretch), 1707 ( $\mathrm{C}=\mathrm{O}$ stretch), 2870, 2960 (aliphatic $\mathrm{C}-\mathrm{H}$ stretch), 3088 (aromatic C-H stretch) $\mathrm{cm}^{-1}$; ${ }^{1} \mathrm{H} \mathrm{NMR}(400 \mathrm{MHz}$, $\left.\mathrm{CDCl}_{3}\right): \delta=1.02\left(6 \mathrm{H}, \mathrm{d}, J=6.64 \mathrm{~Hz},-\mathrm{CH}_{2} \mathrm{CH}\left(\mathrm{CH}_{3}\right)_{2}\right)$, 2.25-2.35 (1H, m, $\left.-\mathrm{CH}_{2} \underline{\mathrm{CH}}\left(\mathrm{CH}_{3}\right)_{2}\right), 2.66(2 \mathrm{H}, \mathrm{d}, J=$ $\left.7.28 \mathrm{~Hz},-\mathrm{CH}_{2} \mathrm{CH}\left(\mathrm{CH}_{3}\right)_{2}\right), 7.36-7.45$ (2H, m, Ar-H), 7.50$7.58(2 \mathrm{H}, \mathrm{m}, \mathrm{Ar}-\mathrm{H}), 7.76(1 \mathrm{H}, \mathrm{d}, J=8.64 \mathrm{~Hz}, \mathrm{Ar}-\mathrm{H}), 7.89$ $(1 \mathrm{H}, \mathrm{d}, J=8.64 \mathrm{~Hz}, \mathrm{Ar}-\mathrm{H}), 8.49$ (1H, d, $J=7.40 \mathrm{~Hz}, 8-\mathrm{H})$; ${ }^{13} \mathrm{C} \mathrm{NMR}\left(100 \mathrm{MHz}, \mathrm{CDCl}_{3}\right): \delta=22.47\left(-\mathrm{CH}_{2} \mathrm{CH}\left(\mathrm{CH}_{3}\right)_{2}\right)$, $27.81\left(-\mathrm{CH}_{2} \underline{\mathrm{CH}}\left(\mathrm{CH}_{3}\right)_{2}\right), 36.34\left(-\mathrm{CH}_{2} \mathrm{CH}\left(\mathrm{CH}_{3}\right)_{2}\right), 121.49$,
$123.85,123.92,124.24,124.37,124.47,130.00,130.46$, $132.55,133.57,134.15,140.09,149.88,150.21,153.04$, 159.80, 184.05 (C-4); HRMS: $\mathrm{m} / \mathrm{z} \quad\left(\mathrm{M}^{+}\right)$Calcd. for $\mathrm{C}_{21} \mathrm{H}_{16} \mathrm{BrN}_{3} \mathrm{OS}$ : 437.0197, found: $438.0257[\mathrm{M}+\mathrm{H}]^{+}$.

\section{Biological studies}

\section{Enzyme assay}

\section{In vitro $a$-glucosidase inhibition}

McCue's protocol was followed for evaluation of in vitro $\alpha$ glucosidase inhibitory activity, with some modifications (McCue et al. 2005). The present activity was carried by using $\alpha$-glucosidase enzyme (Saccharomyces cereviciae). A solution of the enzyme was obtained by adding $20 \mu \mathrm{L} \alpha$ glucosidase $(0.5 \mathrm{unit} / \mathrm{mL})$ in $120 \mu \mathrm{L}$ of $0.1 \mathrm{M}$ phosphate buffer ( $\mathrm{pH}$ 6.9). In microplate wells, the enzyme solution was mixed with $10 \mu \mathrm{L}$ of each test samples which, in turn, were prepared by dissolving in dimethylsulphoxide (DMSO) at various concentrations i.e. 12.5, 25, 50, $100 \mu \mathrm{g} /$ $\mathrm{mL}$ and incubated for $15 \mathrm{~min}$ at $37^{\circ} \mathrm{C}$. Thereafter, this was charged with $20 \mu \mathrm{L}$ of substrate solution to $5 \mathrm{mM}$-nitrophenyl- $\alpha$-D-glucopyranoside in $0.1 \mathrm{M}$ phosphate buffer $(\mathrm{pH}$ 6.9) and further incubated for $15 \mathrm{~min}$. A solution of $0.2 \mathrm{M}$ sodium carbonate $(80 \mu \mathrm{L})$ was added to terminate the reaction, and absorbance was measured at $\lambda=405 \mathrm{~nm}$ on ELISA microplate reader. The reaction system without test samples (4a-0) was used as control while the system without $\alpha$-glucosidase was used as a blank, and Acarbose was used as positive control. Each experiment was performed in triplicate. The enzyme inhibitory rates of samples have been expressed as percentage (\%) inhibition which is determined by Eq. (1) as follows:

$\%$ Inhibition $=\left(\frac{\text { Control Absorption }- \text { Sample Absorption }}{\text { Control Absorption }}\right) \times 100$

The $\mathrm{IC}_{50}$ values of compounds $\mathbf{4 a - 0}$ were calculated.

\section{In vitro $a$-amylase inhibition}

The protocol reported by Xiao et al. and Yoshikawa et al. with slight modifications was utilized for the evaluation of in vitro $\alpha$-amylase inhibition activity (Xiao et al. 2006; Yoshikawa et al. 2001). Stock solutions of compounds $4 \mathbf{a}-\mathbf{0}$ were prepared by dissolving the compound $(5 \mathrm{mg})$ in DMSO $(5 \mathrm{~mL})$ at room temperature. The $\alpha$-amylase inhibitory activity was examined at different concentrations of each sample i.e., $12.5,25,50$, and $100 \mu \mathrm{g} / \mathrm{mL}$. The reagent solution without the test sample was used as the control and 
Acarbose was used as standard reference. Substrate solution was prepared by dissolving soluble starch $(500 \mathrm{mg})$ in $0.4 \mathrm{M} \mathrm{NaOH}(25 \mathrm{~mL})$ and heated for $5 \mathrm{~min}$ at $100^{\circ} \mathrm{C}$. After cooling in ice cold water, the $\mathrm{pH}$ of the solution was achieved to 7 by adding $2 \mathrm{M} \mathrm{HCl}$, and water was added to make the volume to $100 \mathrm{~mL}$. The sample $(20 \mu \mathrm{L})$ and substrate $(40 \mu \mathrm{L})$ solutions were mixed in a microplate well and the mixture in each case was preincubated at $37^{\circ} \mathrm{C}$ for $3 \mathrm{~min}$. Thereafter, $20 \mu \mathrm{L}$ of $\alpha$-amylase solution $(50 \mu \mathrm{g} / \mathrm{mL})$ was added to each well, and the microplate was incubated for $15 \mathrm{~min}$. The reaction was terminated by adding $0.1 \mathrm{M}$ $\mathrm{HCl}(80 \mu \mathrm{L})$. Then $1 \mathrm{mM}$ iodine solution $(200 \mu \mathrm{L})$ was added to the reaction mixture and absorbance was measured at $\lambda=650 \mathrm{~nm}$ with ELISA microplate reader. The enzyme inhibitory activity expressed as percentage (\%) inhibition was calculated by Eq. (2) as follows:

$\%$ Inhibition $=\{1-(A b s 2-A b s 1) /(A b s 4-A b s 3) \times 100\}$

where, Abs1 = Absorbance of incubated solution containing test sample, starch and amylase, Abs2 = Absorbance of incubated solution containing test sample and starch, Abs3 $=$ Absorbance of incubated solution containing starch and amylase, and Abs $4=$ Absorbance of incubated solution containing starch.

\section{In vitro antimicrobial assay}

\section{Test microorganism}

Two Gram-positive bacteria viz. B. subtilis (NCIM 20630) and S. aureus (NCIM 5021), and two Gram-negative bacteria viz. E. coli (MTCC 723) and P. aeruginosa (MTCC 7093), and one fungal strain viz. A. niger (MTCC 9933) were used for antimicrobial assay. All the bacterial cultures were obtained from Microbial Type Culture Collection (MTCC), Institute of Microbial Technology (IMTECH), Chandigarh, India. The nutrient broth utilized for the cultivation of microorganisms was procured from HiMedia Laboratories Pvt. Ltd., Mumbai, India.

\section{Antibacterial evaluation}

All the synthesized compounds $\mathbf{4 a - 0}$ were screened for their antibacterial activity using agar well diffusion method (Okeke et al. 2001). The test microorganisms were enthused by inoculation in $25 \mathrm{~mL}$ of nutrient broth (peptone $5 \mathrm{~g} / \mathrm{L}$, sodium chloride $5 \mathrm{~g} / \mathrm{L}, \mathrm{HM}$ peptone $1.5 \mathrm{~g} /$ $\mathrm{L}$, yeast extract $1.5 \mathrm{~g} / \mathrm{L}, \mathrm{pH}=7.4 \pm 0.2$ ). The media was solidified and the test bacterial strains were cultivated by pour plate method on nutrient agar plates. Wells were bored in the seeded agar plates by using sterile cork borer of $8 \mathrm{~mm}$ diameter and these were loaded with a $100 \mu \mathrm{L}$ of each synthesized compound reconstituted in DMSO. All the plates were incubated at $37^{\circ} \mathrm{C}$ for $24 \mathrm{~h}$. The diameter of inhibition zone of the test organisms was measured by using Digital Colony Counter (Lab and Life Instruments Pvt. Ltd., India) and reported in mm. Sterile DMSO was used as a negative control, whereas Streptomycin was used as a positive control. The experiments were performed in triplicates and the mean values are reported.

\section{Antifungal evaluation}

Antifungal activity of the title compounds 4a-o was examined against $A$. niger by a quantitative microspectrophotometric assay (Broekaert et al. 1990) in 96-well microplates. The growth inhibition was observed at $595 \mathrm{~nm}$. Initially, the fungus was grown on potato dextrose broth (PDB) (Potatoes, infusion form $=200 \mathrm{~g} / \mathrm{L}$, Dextrose $=20 \mathrm{~g} /$ $\mathrm{L}, \mathrm{pH}=5.1 \pm 0.2$ at $25^{\circ} \mathrm{C}$ ) at $27^{\circ} \mathrm{C}$ for 7 days. The spores of the fungus were collected from culture on broth plates. The sporangial suspension concentration was measured by hemocytometer and made to $1.7 \times 10^{5}$ spores $/ \mathrm{mL}$ and the fungal spore suspension was stored at $-40^{\circ} \mathrm{C}$. The PDBfungal spore suspension solution was prepared by mixing PDB $(25 \mathrm{~mL})$ with $0.147 \mathrm{ml}$ of the fungal spore suspension solution. Experiment was performed with $100 \mu \mathrm{L}$ of each of the compounds $\mathbf{4 a - 0}$ to be assayed and $100 \mu \mathrm{L}$ of PDBfungal spore suspension solution. DMSO was used as a negative control and Fluconazole was used as a positive control. After incubation at $27^{\circ} \mathrm{C}$ for $48 \mathrm{~h}$, growth was observed by measuring absorbance at $595 \mathrm{~nm}$ on ELISA plate reader. Growth inhibition was reported by using Eq. (3) given as follows:

$\%$ inhibition $=\left(\frac{\mathrm{A}_{\text {control }}-\mathrm{A}_{\text {sample }}}{\mathrm{A}_{\text {control }}}\right) \times 100$

where $A_{\text {control }}$ is the absorbance of the control and $A_{\text {sample }}$ is the absorbance of the tested microculture.

\section{Experimental protocol of molecular docking study}

Molecular docking analysis was performed using Autodock Vina in Autodock tools (Trott and Olson 2010). For $\alpha$ glucosidase (PDB ID: 5NN5), the coordinates of center of grid box were center_x $=-16.224$, center_y $=-35.668$ and center_z $=95.439$, the size of grid box was $x \_$size $=$ $24 \AA$, y_size $=24 \AA$ and $z \_s i z e=24 \AA$, and the exhaustiveness was equal to 40 . For $\alpha$-amylase (PDB ID: 2QV4), the coordinates of center of grid box were center_x $=20.074$, center_y $=46.866$ and center_z $=$ 29.866 , the size of grid box was $x \_s i z e=28 \AA$, y_size $=$ $28 \AA$ and $\mathrm{z} \_$size $=28 \AA$, and the exhaustiveness was equal to 40 . 
Scheme 1 Synthesis of indeno [1,2-c]pyrazol-4-ones (4a-o)

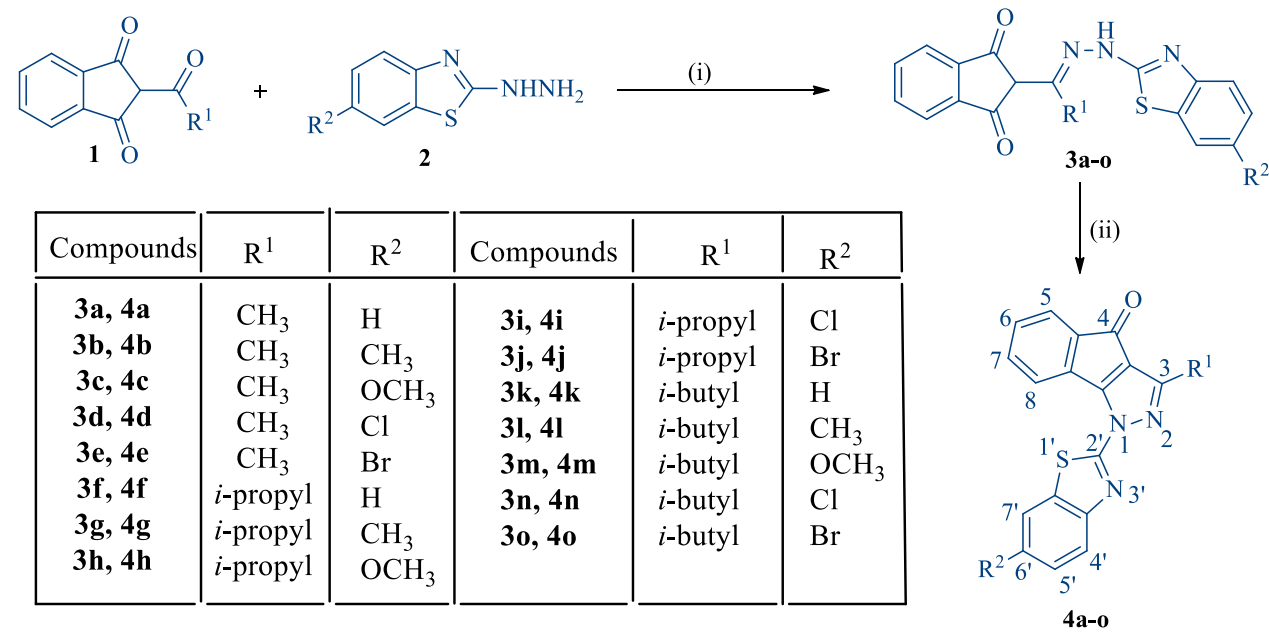

\section{Results and discussion}

\section{Chemistry}

Protocol for the synthesis of some new benzothiazole tethered indeno[1,2-c]pyrazole derivatives (4a-o) is shown in Scheme 1. The chemistry formerly described was utilized for the synthesis of 2-acyl-(1H)-indene-1,3(2H)-diones (1) (Mor et al. 2016). Benzo[d]thiazol-2-amine/6-substitutedbenzo $[d]$ thiazol-2-amines and 2-hydrazinylbenzo $[d]$-thiazole/2-hydrazinyl-6-substitutedbenzo[ $d]$ thiazoles (2) were synthesized by the method reported earlier (Mor et al. 2017). Condensation of appropriate 1,3-diketones (1) with hydrazines (2), in equimolar quantities, in dry methanol under reflux for $15 \mathrm{~min}$ yielded the corresponding benzothiazolyl hydrazones (3) (Mor et al. 2019). The hydrazones (3) thus obtained were subjected to reflux in glacial acetic acid to furnish the target indeno[1,2-c]pyrazol-4-ones $(\mathbf{4 a - 0 )}$ in good yields.

Structures of the newly synthesized compounds 4a-o were confirmed by their FT-IR, NMR $\left({ }^{1} \mathrm{H}\right.$ and $\left.{ }^{13} \mathrm{C}\right)$, and mass spectra. Their FT-IR spectra exhibited strong absorption bands at 1585-1608 $(\mathrm{C}=\mathrm{N})$ and 1693-1716 $(\mathrm{C}=\mathrm{O}) \mathrm{cm}^{-1}$. The main characteristic feature of ${ }^{1} \mathrm{H} \mathrm{NMR}$ spectra of derivatives 4a-o is the resonance of a signal appeared as a doublet integrating for one proton in the range of $\delta 8.03-8.56 \mathrm{ppm},(J=7.28-8.00 \mathrm{~Hz})$, which was safely assigned to $8-\mathrm{H}$. Downfield shifting of this proton is probably due to anisotropic-diamagnetic effect of lone pair of electrons present on nitrogen/sulfur of benzothiazole moiety, which finds support from the results reported earlier (Mor et al. 2016). The significant feature of ${ }^{13} \mathrm{C}$ NMR spectra of compounds 4a-o demonstrated the downfield shifting of signal due to C-4 (carbonyl carbon) which was observed in the region at $\delta 182.64-185.16 \mathrm{ppm}$. However, the remaining protons in ${ }^{1} \mathrm{H}$ NMR and carbons in ${ }^{13} \mathrm{C}$ NMR spectra displayed signals in the expected regions. Further, the HRMS analysis results were found in consistent with their molecular formulae (vide experimental).

\section{Biological studies}

In search of new antidiabetic agents, we recognized primarily various pyrazole derivatives as reported in the literature (Wright et al. 1964). To the best of our knowledge, this is the first report of antidiabetic activity possessed by synthesized indeno[1,2-c]pyrazol-4-ones (4a-o).

\section{In vitro $a$-glucosidase inhibitory activity}

All synthesized compounds $\mathbf{4 a - 0}$ were assessed for their $\alpha$ glucosidase inhibitory activity against $\alpha$-glucosidase enzyme (Saccharomyces cerevisiae) at various concentrations ranging from 12.5 to $100 \mu \mathrm{g} / \mathrm{mL}$ following the developed earlier procedure (McCue et al. 2005) by using Acarbose as the standard (Table 1).

It is inferred from the data presented in Table 1 that all the derivatives exhibited moderate to excellent \% inhibition against $\alpha$-glucosidase enzyme as compared with the standard. Compound $4 \mathbf{i}$ was found to be more potent analogue of this series with $67.02,86.27,90.47$, and $94.52 \%$ inhibition when explored at the concentrations of $12.5,25,50$, and $100 \mu \mathrm{g} / \mathrm{mL}$, respectively. Similarly, compound $4 \mathbf{e}$ exhibited a rise in \% inhibition from 76.41 to $82.76 \%$ on increasing the concentration from 12.5 to $50 \mu \mathrm{g} / \mathrm{mL}$ in comparison to the standard drug Acarbose. Compound $\mathbf{4 l}$ displayed $90.78 \%$ inhibition followed by $\mathbf{4 a}$ that exhibited $87.26 \%$ inhibition at concentration of $100 \mu \mathrm{g} / \mathrm{mL}$. Among the synthesized indeno[1,2-c]pyrazol4-ones, $\mathbf{4 e}$ and $\mathbf{4 i}$ were found to be more active with $\mathrm{IC}_{50}$ values 6.71 and $8.18 \mu \mathrm{g} / \mathrm{mL}$, respectively (Acarbose $\mathrm{IC}_{50}=$ $9.35 \mu \mathrm{g} / \mathrm{mL}$ ). However, the derivatives $\mathbf{4 a}$ and $\mathbf{4 b}$ exhibited good inhibitory activity with $\mathrm{IC}_{50}$ values 9.87 and $10.59 \mu \mathrm{g} /$ $\mathrm{mL}$, respectively. 
Table 1 In vitro $\alpha$-glucosidase inhibitory activities of indeno[1,2-c]pyrazol-4-ones (4a-o)

\begin{tabular}{|c|c|c|c|c|c|c|c|}
\hline \multirow[t]{2}{*}{ Compounds } & \multirow[t]{2}{*}{$\mathrm{R}^{1}$} & \multirow[t]{2}{*}{$\mathrm{R}^{2}$} & \multicolumn{4}{|l|}{$\%$ Inhibition } & \multirow[t]{2}{*}{$\mathrm{IC}_{50}(\mu \mathrm{g} / \mathrm{mL})$} \\
\hline & & & $12.5(\mu \mathrm{g} / \mathrm{mL})$ & $25(\mu \mathrm{g} / \mathrm{mL})$ & $50(\mu \mathrm{g} / \mathrm{mL})$ & $100(\mu \mathrm{g} / \mathrm{mL})$ & \\
\hline $4 \mathbf{a}$ & $\mathrm{CH}_{3}$ & $\mathrm{H}$ & 64.04 & 68.62 & 83.82 & 87.26 & 9.87 \\
\hline $4 b$ & $\mathrm{CH}_{3}$ & $\mathrm{CH}_{3}$ & 61.75 & 71.68 & 76.41 & 77.71 & 10.59 \\
\hline $4 c$ & $\mathrm{CH}_{3}$ & $\mathrm{OCH}_{3}$ & 58.69 & 60.14 & 64.80 & 83.67 & 14.26 \\
\hline $4 d$ & $\mathrm{CH}_{3}$ & $\mathrm{Cl}$ & 60.60 & 62.43 & 63.12 & 65.87 & 15.69 \\
\hline $4 e$ & $\mathrm{CH}_{3}$ & $\mathrm{Br}$ & 76.41 & 82.76 & 86.04 & 86.12 & 6.71 \\
\hline $4 f$ & $\mathrm{CH}\left(\mathrm{CH}_{3}\right)_{2}$ & $\mathrm{H}$ & 35.48 & 43.00 & 58.02 & 80.12 & 29.78 \\
\hline $4 g$ & $\mathrm{CH}\left(\mathrm{CH}_{3}\right)_{2}$ & $\mathrm{CH}_{3}$ & 49.45 & 54.34 & 57.55 & 59.07 & 26.76 \\
\hline $4 h$ & $\mathrm{CH}\left(\mathrm{CH}_{3}\right)_{2}$ & $\mathrm{OCH}_{3}$ & 45.63 & 47.08 & 53.50 & 64.50 & 31.66 \\
\hline $4 i$ & $\mathrm{CH}\left(\mathrm{CH}_{3}\right)_{2}$ & $\mathrm{Cl}$ & 67.02 & 86.27 & 90.47 & 94.52 & 8.18 \\
\hline $4 j$ & $\mathrm{CH}\left(\mathrm{CH}_{3}\right)_{2}$ & $\mathrm{Br}$ & 35.25 & 47.87 & 69.34 & 86.12 & 24.03 \\
\hline $4 k$ & $\mathrm{CH}_{2} \mathrm{CH}\left(\mathrm{CH}_{3}\right)_{2}$ & $\mathrm{H}$ & 55.94 & 68.01 & 68.62 & 71.30 & 13.83 \\
\hline 41 & $\mathrm{CH}_{2} \mathrm{CH}\left(\mathrm{CH}_{3}\right)_{2}$ & $\mathrm{CH}_{3}$ & 62.66 & 65.87 & 84.82 & 90.78 & 10.41 \\
\hline $4 m$ & $\mathrm{CH}_{2} \mathrm{CH}\left(\mathrm{CH}_{3}\right)_{2}$ & $\mathrm{OCH}_{3}$ & 46.93 & 51.74 & 51.97 & 57.85 & 33.49 \\
\hline $4 n$ & $\mathrm{CH}_{2} \mathrm{CH}\left(\mathrm{CH}_{3}\right)_{2}$ & $\mathrm{Cl}$ & 42.57 & 58.16 & 69.00 & 83.98 & 18.83 \\
\hline 40 & $\mathrm{CH}_{2} \mathrm{CH}\left(\mathrm{CH}_{3}\right)_{2}$ & $\mathrm{Br}$ & 53.57 & 60.91 & 64.57 & 78.40 & 16.03 \\
\hline Acarbose & - & - & 61.98 & 78.86 & 87.64 & 94.06 & 9.35 \\
\hline
\end{tabular}

Table 2 In vitro $\alpha$-amylase inhibitory activity of indeno[1,2-c]pyrazol-4-ones (4a-o)

\begin{tabular}{|c|c|c|c|c|c|c|c|}
\hline \multirow[t]{2}{*}{ Compounds } & \multirow[t]{2}{*}{$\mathrm{R}^{1}$} & \multirow[t]{2}{*}{$\mathrm{R}^{2}$} & \multicolumn{4}{|l|}{$\%$ Inhibition } & \multirow[t]{2}{*}{$\mathrm{IC}_{50}(\mu \mathrm{g} / \mathrm{mL})$} \\
\hline & & & $12.5(\mu \mathrm{g} / \mathrm{mL})$ & $25(\mu \mathrm{g} / \mathrm{mL})$ & $50(\mu \mathrm{g} / \mathrm{mL})$ & $100(\mu \mathrm{g} / \mathrm{mL})$ & \\
\hline $4 a$ & $\mathrm{CH}_{3}$ & $\mathrm{H}$ & 36.42 & 47.14 & 85.00 & 89.28 & 21.23 \\
\hline $4 b$ & $\mathrm{CH}_{3}$ & $\mathrm{CH}_{3}$ & 7.14 & 15.71 & 70.71 & 82.14 & 40.46 \\
\hline $4 c$ & $\mathrm{CH}_{3}$ & $\mathrm{OCH}_{3}$ & 28.57 & 37.14 & 63.57 & 87.14 & 31.28 \\
\hline $4 d$ & $\mathrm{CH}_{3}$ & $\mathrm{Cl}$ & 22.14 & 50.71 & 72.14 & 85.71 & 26.44 \\
\hline $4 e$ & $\mathrm{CH}_{3}$ & $\mathrm{Br}$ & 37.85 & 59.28 & 73.57 & 86.42 & 19.25 \\
\hline $4 f$ & $\mathrm{CH}\left(\mathrm{CH}_{3}\right)_{2}$ & $\mathrm{H}$ & 19.28 & 55.00 & 60.00 & 77.14 & 30.61 \\
\hline $4 \mathrm{~g}$ & $\mathrm{CH}\left(\mathrm{CH}_{3}\right)_{2}$ & $\mathrm{CH}_{3}$ & 15.714 & 31.42 & 41.42 & 58.57 & 68.36 \\
\hline $4 h$ & $\mathrm{CH}\left(\mathrm{CH}_{3}\right)_{2}$ & $\mathrm{OCH}_{3}$ & 22.85 & 36.42 & 65.71 & 72.85 & 35.8 \\
\hline $4 \mathbf{i}$ & $\mathrm{CH}\left(\mathrm{CH}_{3}\right)_{2}$ & $\mathrm{Cl}$ & 57.85 & 67.14 & 73.57 & 92.14 & 11.90 \\
\hline $4 \mathbf{j}$ & $\mathrm{CH}\left(\mathrm{CH}_{3}\right)_{2}$ & $\mathrm{Br}$ & 28.57 & 39.28 & 50.71 & 81.42 & 36.44 \\
\hline $4 k$ & $\mathrm{CH}_{2} \mathrm{CH}\left(\mathrm{CH}_{3}\right)_{2}$ & $\mathrm{H}$ & 16.42 & 31.42 & 66.42 & 77.14 & 37.62 \\
\hline 41 & $\mathrm{CH}_{2} \mathrm{CH}\left(\mathrm{CH}_{3}\right)_{2}$ & $\mathrm{CH}_{3}$ & 31.42 & 55.71 & 67.14 & 82.14 & 23.71 \\
\hline $4 m$ & $\mathrm{CH}_{2} \mathrm{CH}\left(\mathrm{CH}_{3}\right)_{2}$ & $\mathrm{OCH}_{3}$ & 25.71 & 40.71 & 57.85 & 83.57 & 33.36 \\
\hline $4 n$ & $\mathrm{CH}_{2} \mathrm{CH}\left(\mathrm{CH}_{3}\right)_{2}$ & $\mathrm{Cl}$ & 24.28 & 45.71 & 61.42 & 73.57 & 33.14 \\
\hline 40 & $\mathrm{CH}_{2} \mathrm{CH}\left(\mathrm{CH}_{3}\right)_{2}$ & $\mathrm{Br}$ & 54.28 & 65.71 & 78.57 & 82.14 & 12.83 \\
\hline Acarbose & - & - & 30.71 & 51.42 & 75.71 & 92.14 & 22.87 \\
\hline
\end{tabular}

\section{In vitro $a$-amylase inhibitory activity}

The $\alpha$-amylase inhibitory activity of the synthesized compounds 4a-0 was screened following Xiao's procedure (Xiao et al. 2006; Yoshikawa et al. 2001) by using Acarbose as the standard reference (Table 2).

The results of the $\alpha$-amylase inhibitory activity depicted in Table 2 revealed that all the tested derivatives $\mathbf{4 a - 0}$ displayed moderate to high \% inhibition. Compounds $\mathbf{4 a}, \mathbf{4 e}$, $\mathbf{4 i}, 4 \mathbf{l}$, and $\mathbf{4 o}$ at concentration $12.5 \mu \mathrm{g} / \mathrm{mL}, \mathbf{4 e}, \mathbf{4 f}, 4 \mathbf{i}, \mathbf{4 l}$, and 40 at concentration $25 \mu \mathrm{g} / \mathrm{mL}$, and $\mathbf{4 a}$ and $4 \mathbf{o}$ at concentration $50 \mu \mathrm{g} / \mathrm{mL}$ were found to be more potent than the standard. Whereas compound $\mathbf{4 i}$ displayed inhibition equivalent to the standard drug Acarbose at concentration $100 \mu \mathrm{g} / \mathrm{mL}$. The analogues $\mathbf{4 c}$ and $\mathbf{4 j}$ at concentration $12.5 \mu \mathrm{g} / \mathrm{mL}, \mathbf{4 a}$ and $\mathbf{4 d}$ at concentration $25 \mu \mathrm{g} / \mathrm{mL}, \mathbf{4 b}, \mathbf{4 d}, \mathbf{4 e}$, and $\mathbf{4 i}$ at concentration $50 \mu \mathrm{g} / \mathrm{mL}$, and $\mathbf{4 a}$ and $\mathbf{4 c}$ at concentration 
$100 \mu \mathrm{g} / \mathrm{mL}$ demonstrated comparable inhibitory activity as shown by the standard drug Acarbose. Furthermore, the remaining compounds were found to display lesser inhibitory activity as compared with the standard drug screened at different concentrations. Among the synthesized derivatives, $\mathbf{4 a}\left(\mathrm{IC}_{50}=\right.$ $21.23 \mu \mathrm{g} / \mathrm{mL}), \mathbf{4 e}\left(\mathrm{IC}_{50}=19.25 \mu \mathrm{g} / \mathrm{mL}\right), \mathbf{4 i}\left(\mathrm{IC}_{50}=11.90 \mu \mathrm{g} /\right.$ $\mathrm{mL})$, and $40\left(\mathrm{IC}_{50}=12.83 \mu \mathrm{g} / \mathrm{mL}\right)$ demonstrated higher activity than the standard (Acarbose, $\mathrm{IC}_{50}=22.87 \mu \mathrm{g} / \mathrm{mL}$ ). Consequently, $\mathbf{4 a}, \mathbf{4 e}, \mathbf{4 i}$, and 40 can be considered as a possible antidiabetic agent for further studies.

\section{Structure activity relationship (SAR) for antidiabetic activity of indeno[1,2-c]pyrazol-4-ones (4a-0)}

According to the presented data for antidiabetic activity of indeno[1,2-c]pyrazol-4-ones (4a-o), the following SARs have been established:

(1) Results of antidiabetic activity indicated that the presence of $\mathrm{R}^{1}=\mathrm{CH}_{3}$ and $\mathrm{R}^{2}=\mathrm{H}, \mathrm{Br}$ in the synthesized compounds $\mathbf{4 a - 0}$ has led to increase the antidiabetic activity against $\alpha$-glucosidase enzyme while the derivative $\mathbf{4 e}$ containing $\mathrm{R}^{1}=\mathrm{CH}_{3}$ and $\mathrm{R}^{2}$ $=\mathrm{Br}$ has been found to exhibit improved inhibitory activity against $\alpha$-amylase enzyme.

(2) Compound $4 \mathbf{i}$ containing $\mathrm{R}^{1}=i$-propyl and $\mathrm{R}^{2}=\mathrm{Cl}$ has been found to enhance inhibitory activity against both $\alpha$-glucosidase and $\alpha$-amylase enzymes.

(3) Derivative $4 \mathbf{l}$ containing $\mathrm{R}^{1}=i$-butyl and $\mathrm{R}^{2}=\mathrm{CH}_{3}$ has improved inhibitory activity against $\alpha$-glucosidase, whereas presence of $\mathrm{R}^{1}=i$-butyl and $\mathrm{R}^{2}=\mathrm{Br}$ in compound 40 had increased inhibitory activity against $\alpha$-amylase enzyme.

From these results, it is inferred that the compounds containing $\mathrm{R}^{1}=\mathrm{CH}_{3}$ and $\mathrm{R}^{2}=\mathrm{H}, \mathrm{CH}_{3}, \mathrm{OCH}_{3}, \mathrm{Cl}$, and $\mathrm{Br}$, are found to display more inhibitory activity against $\alpha$ glucosidase as compared with the remaining derivatives containing $\mathrm{R}^{1}=i$-propyl and $i$-butyl, and $\mathrm{R}^{2}=\mathrm{H}, \mathrm{CH}_{3}$, $\mathrm{OCH}_{3}, \mathrm{Cl}$, and $\mathrm{Br}$, whereas no such trend was observed against $\alpha$-amylase inhibitory activity. Overall, we may conclude that there are different structural requirements for a compound to be effective against $\alpha$-glucosidase and $\alpha$ amylase enzymes. However, no general trend for SAR was established for both $\alpha$-glucosidase and $\alpha$-amylase enzymes. The above mentioned findings are summarized in Fig. 1.

\section{In vitro antibacterial activity}

All synthesized indeno[1,2-c]pyrazol-4-ones (4a-o) were tested for their in vitro antibacterial activity against Grampositive (B. subtilis, S. aureus) and Gram-negative (E. coli, $P$. aeruginosa) bacteria by agar well diffusion method using Streptomycin as the reference drug (Table 3) (Okeke et al. 2001).

It is revealed from the data presented in Table 3 that compound $\mathbf{4 l}$ and $\mathbf{4 g}$ exhibited the highest activity against $B$. subtilis, while the derivative $\mathbf{4 h}$ demonstrated maximum inhibition zone against $S$. aureus. Moreover, the analogues $\mathbf{4 f}$ and $\mathbf{4 j}$ displayed moderate activity against $E$. coli, while $\mathbf{4 g}$ demonstrated activity against $P$. aeruginosa. On the other hand, some compounds were inactive against the specific bacteria under study. Overall, these results suggest that the synthesized compounds 4a-o exhibit lesser activity against the bacterial strains than standard drug Streptomycin.

\section{In vitro antifungal activity}

All synthesized compounds $\mathbf{4 a - 0}$ were evaluated for their in vitro antifungal activity against $A$. niger by the quantitative microspectrophotometric assay using Fluconazole as the standard drug (Broekaert et al. 1990) (Table 4).

A perusal of accumulated data from Table 4 reveals that all synthesized compounds $\mathbf{4 a - 0}$ were found to inhibit fungal growth with inhibition ranging from 49.21 to $72.25 \%, 54.71$ to $76.96 \%, 59.42$ to $80.89 \%$, and 67.01 to $84.55 \%$ at concentration $125,250,500$, and $1000 \mu \mathrm{g} / \mathrm{mL}$, respectively. Derivative 40 was found to be more active with $\mathrm{IC}_{50}$ value of $5.68 \mu \mathrm{g} / \mathrm{mL}$ than the standard $\left(\mathrm{IC}_{50}=\right.$
Fig. 1 Structure activity relationship (SAR) for antidiabetic activity of synthesized indeno[1,2-c] pyrazol-4-ones (4a-o)
The analogue containing $\mathrm{R}^{1}=i$-butyl and $\mathrm{R}^{2}=\mathrm{CH}_{3}$ improved inhibitory activity against $a$-glucosidase, and the compound containing $\mathrm{R}^{1}=i$-butyl and $\mathrm{R}^{2}=\mathrm{Br}$ increased inhibitory activity against $a$-amylase enzyme

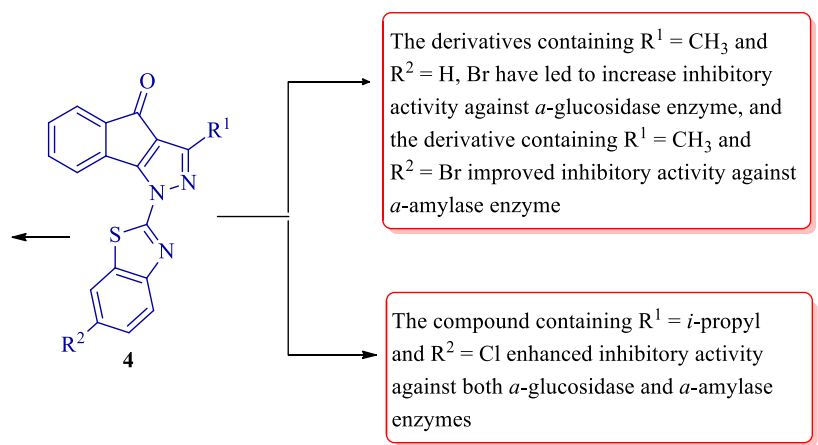




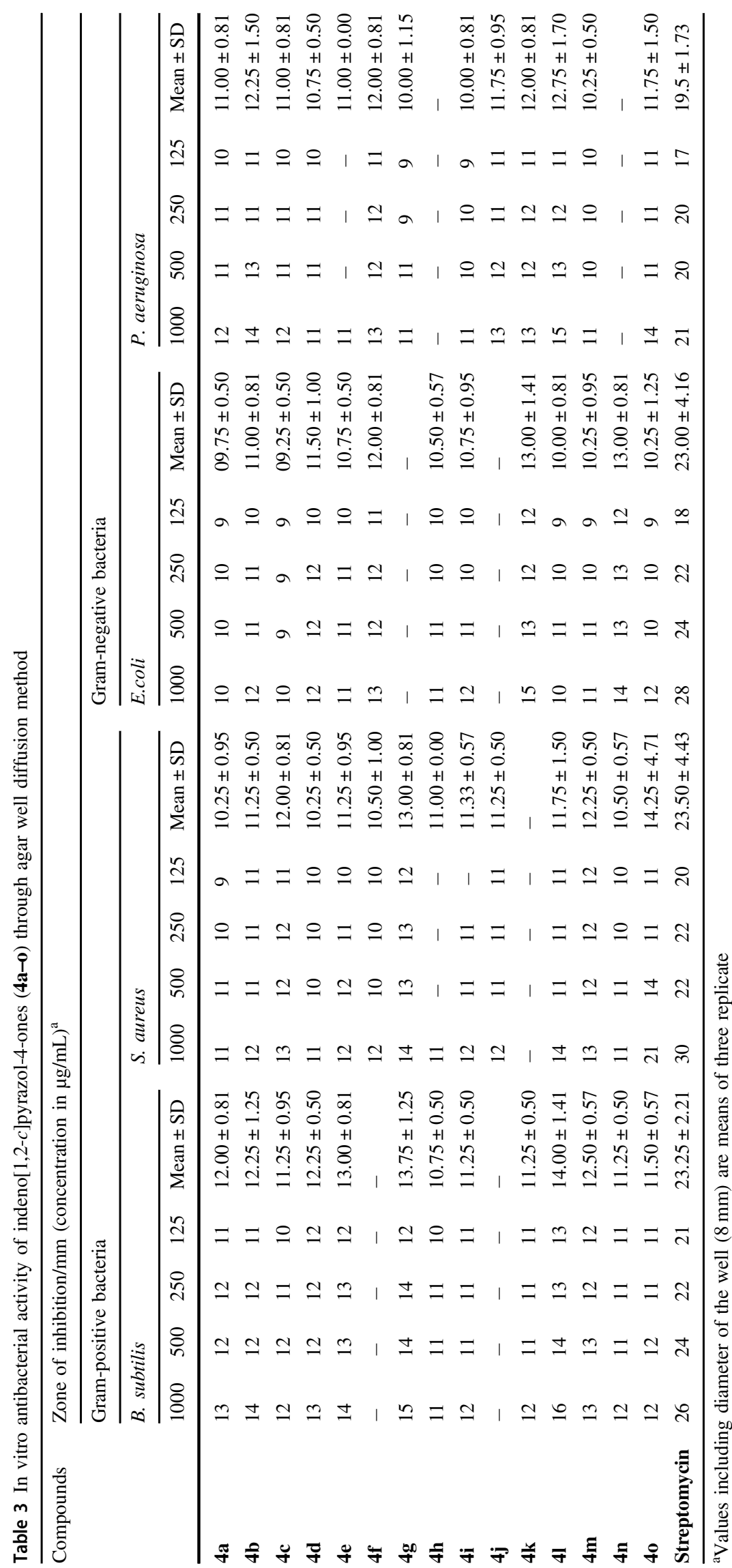


$12.73 \mu \mathrm{g} / \mathrm{mL})$. Compounds $\mathbf{4 d}, \mathbf{4 f}, \mathbf{4 h}$, and $\mathbf{4 n}$ demonstrated comparable activity to standard drug Fluconazole. Overall, these results suggest that the synthesized compounds $4 \mathbf{a}-\mathbf{o}$ demonstrated good activity at different concentrations against the fungal strain.

\section{SARs for antimicrobial activity of indeno[1,2-c]pyrazol-4- ones $(4 a-0)$}

The SARs approach to the synthesized derivatives $4 \mathbf{a}-\mathbf{o}$ demonstrated good to moderate inhibitory potential against all the microbial strains under study. Consequently, we may conclude that there are different structural requirements for a compound to be effective against different bacterial and fungal strains. Moreover, no general trend has been established for the SARs for antimicrobial activity.

\section{Molecular docking analysis}

Molecular docking analysis of some selected synthesized compounds with enzymes $\alpha$-glucosidase and $\alpha$-amylase was

Table 4 In vitro antifungal activity of indeno[1,2-c]pyrazol-4-ones (4a-o) against $A$. niger in terms of $\%$ inhibition and $\mathrm{IC}_{50}$ values

\begin{tabular}{|c|c|c|c|c|c|}
\hline \multirow[t]{2}{*}{ Compounds } & \multicolumn{4}{|l|}{$\%$ Inhibition } & \multirow[t]{2}{*}{$\mathrm{IC}_{50}(\mu \mathrm{g} / \mathrm{mL})$} \\
\hline & $125(\mu \mathrm{g} / \mathrm{mL})$ & $250(\mu \mathrm{g} / \mathrm{mL})$ & $500(\mu \mathrm{g} / \mathrm{mL})$ & $1000(\mu \mathrm{g} / \mathrm{mL})$ & \\
\hline $4 \mathbf{a}$ & 55.23 & 59.94 & 66.75 & 70.68 & 60.25 \\
\hline $4 b$ & 49.47 & 58.37 & 65.96 & 71.20 & 154.54 \\
\hline $4 c$ & 49.21 & 54.71 & 60.73 & 67.01 & 130.31 \\
\hline $4 d$ & 64.13 & 69.89 & 74.34 & 78.27 & 17.58 \\
\hline $4 e$ & 54.71 & 63.61 & 65.96 & 76.70 & 79.43 \\
\hline $4 f$ & 69.63 & 74.34 & 80.89 & 84.55 & 14.28 \\
\hline $4 g$ & 59.68 & 71.98 & 78.79 & 83.76 & 45.70 \\
\hline $4 h$ & 69.63 & 74.34 & 80.89 & 84.55 & 14.29 \\
\hline $4 i$ & 64.13 & 73.56 & 78.79 & 81.93 & 25.70 \\
\hline $4 j$ & 54.97 & 63.35 & 72.77 & 75.65 & 74.13 \\
\hline $4 k$ & 63.08 & 65.96 & 73.29 & 80.62 & 55.59 \\
\hline 41 & 51.30 & 57.32 & 59.42 & 67.01 & 100.00 \\
\hline $4 m$ & 57.32 & 66.23 & 72.25 & 76.96 & 48.97 \\
\hline $4 n$ & 63.87 & 70.68 & 74.86 & 78.27 & 18.62 \\
\hline 40 & 72.25 & 76.96 & 79.31 & 82.98 & 05.68 \\
\hline Fluconazole & 74.08 & 85.60 & 89.73 & 92.40 & 12.73 \\
\hline
\end{tabular}

Fig. 2 Docked pose of compound 4e (green) showing three pi-pi interactions in blue color with amino acid residue PHE-525, TRP-376 (T shaped), and TRP-481 (T shaped) of $\alpha$ glucosidase

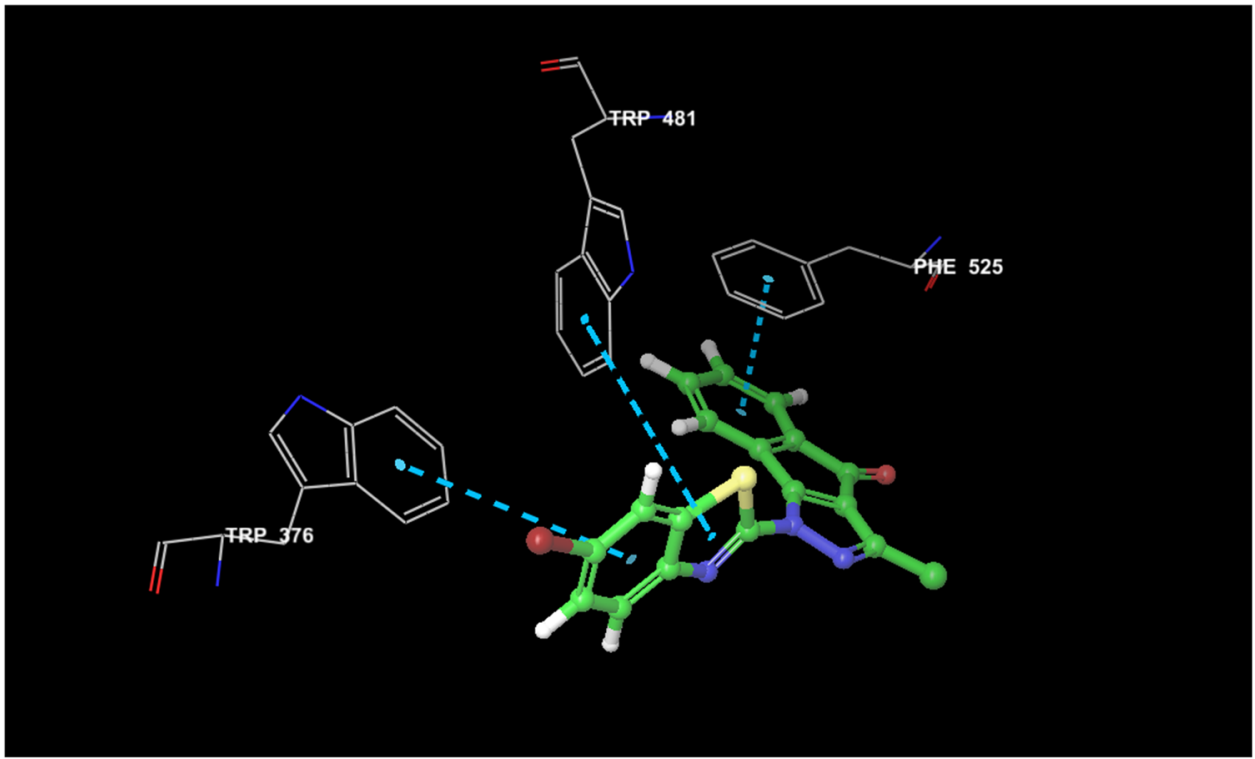


Fig. 3 Docked pose of compound $\mathbf{4 i}$ (grey) inside the active site of $\alpha$-glucosidase showing three pi-pi interactions in blue color with amino acid residue PHE-525, TRP-376 (T shaped), and TRP-481 (T shaped)
Fig. 4 Docked pose of compound 40 (white) showing two hydrogen bonding interactions (yellow) with GLN63 and THR-163 and three Pi-Pi interactions (blue) with TRP-59 of $\alpha$-amylase
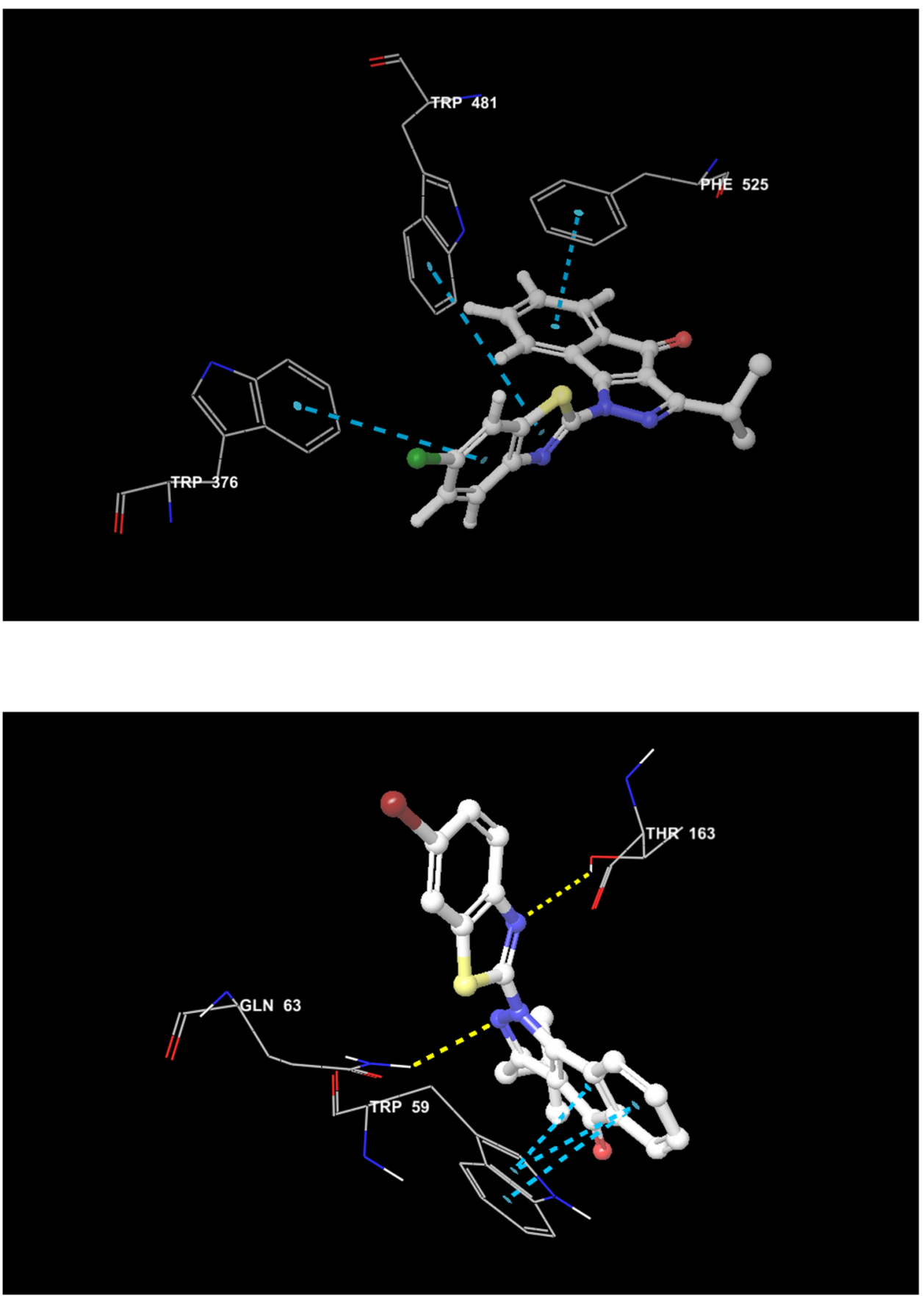

performed to find out the mechanism of action at the molecular level.

\section{Molecular docking with $a$-glucosidase}

Crystal structure used for $\alpha$-glucosidase was obtained from protein data bank and the PDB ID for this structure is 5NN5 (Roig-Zamboni et al. 2017). Docking protocol was validated by docking the co-crystallized ligand. In vitro assay showed that compounds $\mathbf{4 e}$ and $\mathbf{4 i}$ exhibit best $\alpha$-glucosidase inhibitory potential, hence molecular docking analysis was performed using these two compounds. Compound $\mathbf{4 e}$ with a docking score of -7.3 showed three pi-pi interactions with amino acid residue PHE-525, TRP-376 (T shaped), and TRP-481 (T shaped) as shown in Fig. 2 and hydrophobic interactions with TRP-481 and PHE-649. Compound $4 \mathbf{i}$ with docking score of -8.4 showed interactions very similar to that of $\mathbf{4 e}$ i.e., three pi-pi interactions with amino acid residue PHE-525, TRP-376 (T shaped), and TRP-481 ( $\mathrm{T}$ shaped) as shown in Fig. 3 and further stabilizing interactions are provided by hydrophobic contacts with ASP-282, ALA-555, and PHE-649. Both the 
Fig. 5 Docked pose of compound $4 \mathbf{i}$ (green) showing one hydrogen bond with GLN 63 (pink), one halogen bond with ASP 197 (violet), and 3 pi-pi interactions with TRP 59 (blue) of $\alpha$-amylase

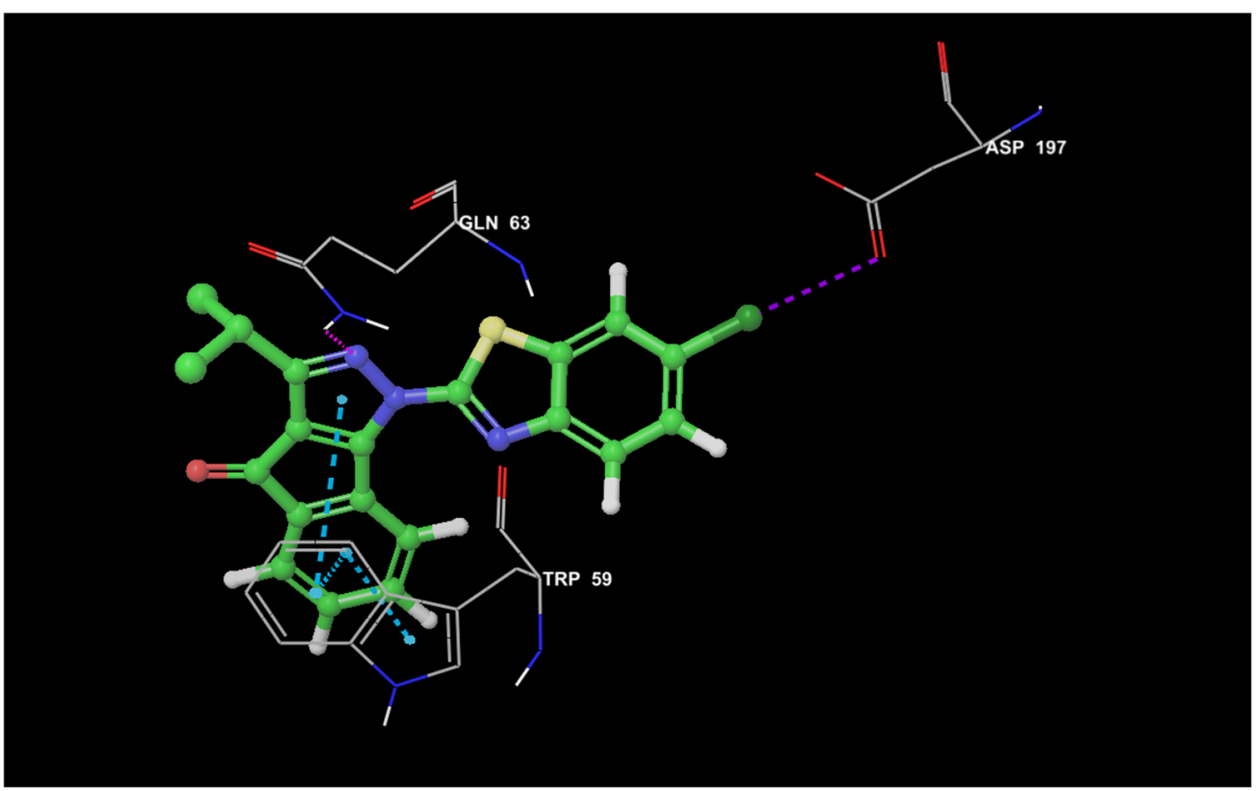

compounds were found to bind in similar orientation with a snug fit.

\section{Molecular docking with a-amylase}

In vitro results showed that most of the synthesized compounds 4a-o were stronger inhibitors of $\alpha$-amylase compared with acarbose. Compounds $4 \mathbf{i}$ and 40 showed superior inhibition as compared with the other synthesized compounds. Therefore, $\mathbf{4 i}$ and $\mathbf{4 0}$ compounds were used for determining the binding pose and interactions responsible for the activity against human pancreatic $\alpha$-amylase (PDB ID: 2QV4) (Maurus et al. 2008). First docking protocol was validated by performing docking of co-crystallized ligand. Compound 40 with docking score of -8.8 was found to be showing two hydrogen bond interactions with amino acid residues GLN-63 and THR-163, three pi-pi interactions with TRP-59 and hydrophobic contacts with TYR-62, LEU162, and LEU-165. The binding pose of compound 4o is shown in Fig. 4. Compound $\mathbf{4 i}$ with docking score of -9.3 binds in active site of $\alpha$-amylase with orientation and interactions similar to that of $\mathbf{4 o}$. It is showing one hydrogen bond with GLN-63, one halogen bond with ASP-197, three pi-pi interactions with TRP-59 and hydrophobic contacts with TRP-58 and TYR-62 as shown in Fig. 5.

\section{Conclusion}

In conclusion, the present study describe the synthesis and characterization of heterocyclic frameworks i.e., indeno [1,2-c]pyrazoles (4), and their biological evaluation as inhibitor of $\alpha$-glucosidase and $\alpha$-amylase related to Type II diabetes, and antimicrobial activity. The chemistry for the synthesis of indeno[1,2-c]pyrazole (4) involved the reaction of 2-acyl-(1H)-indene-1,3(2H)-diones (1) with 2-hydrazinylbenzo $[d]$ thiazole/2-hydrazinyl-6-substitutedbenzo[ $d]$ thiazoles (2) in dry methanol to give benzothiazolyl hydrazones (3), which upon subsequent refluxing in glacial acetic acid afforded the target compounds $\mathbf{4}$ in good yields. Some of the synthesized compounds exhibited significant in vitro $\alpha$ glucosidase and $\alpha$-amylase inhibitory activity viz. 4e was found to be more potent with $\mathrm{IC}_{50}$ value $6.71 \mu \mathrm{g} / \mathrm{mL}$ against $\alpha$-glucosidase enzyme and $\mathbf{4} \mathbf{i}$ showed good activity with $\mathrm{IC}_{50}$ value $11.90 \mu \mathrm{g} / \mathrm{mL}$ against $\alpha$-amylase enzyme as compared with reference drug Acarbose $\left(\mathrm{IC}_{50}=22.87 \mu \mathrm{g} / \mathrm{mL}\right)$. Moreover, some of the compounds exhibited convincing results for antimicrobial activity, however, with a degree of variation. The antidiabetic activity was found to be more prolific than antimicrobial activity. In vitro $\alpha$-glucosidase and $\alpha$ amylase inhibition was further supported by docking studies of compounds $\mathbf{4 e}, \mathbf{4 i}$, and $\mathbf{4 o}$. Hopefully, these findings will prove helpful to medicinal chemists for the development of new inhibitors of enzymes related to Type II diabetes.

Acknowledgements The authors are grateful to the Council of Scientific and Industrial Research, New Delhi, India for providing financial support (CSIR No. 09/752(0060)/2016-EMR-I).

\section{Compliance with ethical standards}

Conflict of interest The authors declare that they have no conflict of interest. 
Publisher's note Springer Nature remains neutral with regard to jurisdictional claims in published maps and institutional affiliations.

\section{References}

Ahsan MJ, Samy JG, Soni S, Jain N, Kumar L, Sharma LK, Prasad R (2011) Discovery of novel antitubercular 3a,4-dihydro- $3 H$ indeno[1,2-c]pyrazole-2-carboxamide/carbothioamide analogues. Bioorg Med Chem Lett 21(18):5259-5261

Ahsan MJ, Govindasamy J, Khalilullah H, Mohan G, Stables JP, Pannecouque C, De Clercq E (2012) POMA analyses as new efficient bioinformatics platform to predict and optimise bioactivity of synthesized 3a,4-dihydro- $3 H$-indeno[1,2-c]pyrazole-2carboxamide/carbothioamide analogues. Bioorg Med Chem Lett 22(23):7029-7035

Ajiboye BO, Ojo OA, Adeyonu O, Imiere O, Olayide I, Fadaka A, Oyinloye BE (2016) Inhibitory effect on key enzymes relevant to acute type- 2 diabetes and antioxidative activity of ethanolic extract of artocarpus heterophyllus stem bark. J Acute Dis 5 (5):423-429

Amnerkar ND, Bhusari KP (2010) Synthesis, anticonvulsant activity and 3D-QSAR study of some prop-2-eneamido and 1-acetylpyrazolin derivatives of aminobenzothiazole. Eur J Med Chem 45 (1):149-159

Bhat M, Belagali SL (2016) Guanidinyl benzothiazole derivatives: Synthesis and structure activity relationship studies of a novel series of potential antimicrobial and antioxidants. Res Chem Intermed 42(7):6195-6208

Broekaert WF, Terras FRG, Cammue BPA, Vanderleyden J (1990) An automated quantitative assay for fungal growth inhibition. FEMS Microbiol Lett 69:55-60

Carvalho VF, Barreto EO, Serra MF, Cordeiro RS, Martins MA, Fortes ZB, e Silva PM (2006) Aldose reductase inhibitor zopolrestat restores allergic hyporesponsiveness in alloxan-diabetic rats. Eur J Pharm 549(1):173-178

Chaudhry F, Naureen S, Huma R, Shaukat A, al-Rashida M, Asif N, Khan MA (2017) In search of new $\alpha$-glucosidase inhibitors: Imidazolylpyrazole derivatives. Bioorg Chem 71:102-109

Chugunova E, Boga C, Sazykin I, Cino S, Micheletti G, Mazzanti A, Kostina N (2015) Synthesis and antimicrobial activity of novel structural hybrids of benzofuroxan and benzothiazole derivatives. Eur J Med Chem 93:349-359

Doddaramappa SD, Rai KL, Srikantamurthy N, Chethan J (2015) Novel 5-functionalized-pyrazoles: synthesis, characterization and pharmacological screening. Bioorg Med Chem Lett 25 (17):3671-3675

Gabr MT, El-Gohary NS, El-Bendary ER, El-Kerdawy MM (2015) New series of benzothiazole and pyrimido[2,1-b]benzothiazole derivatives: synthesis, antitumor activity, EGFR tyrosine kinase inhibitory activity and molecular modeling studies. Med Chem Res 24(2):860-878

Huang G, Huang H (2019) Synthesis, antiasthmatic, and insecticidal/ antifungal activities of allosamidins. J Enzym Inhib Med Chem 34(1):1226-1232

International Diabetes Foundation (2017) IDF diabetes atlas. 8th ed. International Diabetes Foundation, Brussels

Kamal A, Syed MAH, Mohammed SM (2015) Therapeutic potential of benzothiazoles: a patent review (2010-2014). Expert Opin Ther Pat 25(3):335-349

Khan I, Garikapati KR, Setti A, Shaik AB, Makani VKK, Shareef MA, Kumar CG (2019) Design, synthesis, in silico pharmacokinetics prediction and biological evaluation of 1,4-dihydroindeno[1,2-c] pyrazole chalcone as EGFR/Akt pathway inhibitors. Eur J Med Chem 163:636-648
Khan I, Shareef MA, Kumar CG (2019) An overview on the synthetic and medicinal perspectives of indenopyrazoles Eur J Med Chem 178:1-12

Keri RS, Patil MR, Patil SA, Budagumpi S (2015) A comprehensive review in current developments of benzothiazole-based molecules in medicinal chemistry. Eur J Med Chem 89:207-251

Kim MB, O'Brien TE, Moore JT, Anderson DE, Foss MH, Weibel DB, Shaw JT (2012) The synthesis and antimicrobial activity of heterocyclic derivatives of totarol. ACS Med Chem Lett 3(10):818-822

Kumar P, Duhan M, Kadyan K, Sindhu J, Kumar S, Sharma H (2017) Synthesis of novel inhibitors of $\alpha$-amylase based on the thiazolidine-4-one skeleton containing a pyrazole moiety and their configurational studies. Med Chem Comm 8(7):1468-1476

Lemke TL, Abebe E, Moore PF, Carty TJ (1989) Indeno [1,2-c] pyrazolone acetic acids as semirigid analogues of the nonsteroidal anti-inflammatory drugs. J Pharm Sci 78(4):343-347

Lemke TL, Cramer MB, Shanmugam K (1978) Heterocyclic tricycles as potential CNS agents I: 4-aminoalkylindeno [1,2-c] pyrazoles. J Pharm Sci 67(10):1377-1381

Maurus R, Begum A, Williams LK, Fredriksen JR, Zhang R, Withers SG, Brayer GD (2008) Alternative catalytic anions differentially modulate human $\alpha$-amylase activity and specificity. Biochemistry 47(11):3332-3344

McCue P, Kwon Y-I, Shetty K (2005) Anti-amylase, anti-glucosidase and anti-angiotensin I-converting enzyme potential of selected foods. J Food Biochem 29(3):278-294

Meltzer-Mats E, Babai-Shani G, Pasternak L, Uritsky N, Getter T, Viskind O, Gruzman A (2013) Synthesis and mechanism of hypoglycemic activity of benzothiazole derivatives. J Med Chem 56(13):5335-5350

Minegishi H, Futamura Y, Fukashiro S, Muroi M, Kawatani M, Osada H, Nakamura H (2015) Methyl 3-((6-methoxy-1,4-dihydroindeno [1,2-c]pyrazol-3-yl)amino) benzoate (GN39482) as a tubulin polymerization inhibitor identified by MorphoBase and ChemProteoBase profiling methods. J Med Chem 58(10):4230-4241

Mor S, Nagoria S, Kumar A, Monga J, Lohan S (2016) Convenient synthesis, anticancer evaluation and QSAR studies of some thiazole tethered indenopyrazoles. Med Chem Res 25(6):1096-1114

Mor S, Mohil R, Nagoria S, Kumar A, Lal K, Kumar D, Singh V (2017) Regioselective synthesis, antimicrobial evaluation and QSAR studies of some 3-aryl-1-heteroarylindeno [1,2-c]pyrazol$4(1 H)$-ones. J Heterocycl Chem 54(2):1327-1341

Mor S, Nagoria S, Sindhu S, Khatri M, Sidhu G, Singh V (2017) Synthesis of indane-Based 1,5-benzothiazepines derived from 3Phenyl-2,3-dihydro- $1 H$-inden-1-one and antimicrobial studies thereof. J Heterocycl Chem 54(6):3282-3293

Mor S, Sindhu S, Nagoria S, Khatri M, Garg P, Sandhu H, Kumar A (2019) Synthesis, biological evaluation, and molecular docking studies of some $N$-thiazolyl hydrazones and indenopyrazolones. J Heterocycl Chem 56(5):1622-1633

Mor S, Sindhu S, Khatri M, Singh N, Vasudeva N, Panihar N (2019) Synthesis, type II Diabetes inhibitory activity, and antimicrobial tests of benzothiazole derivatives bridged with indenedione by methylenehydrazone. Russ J Gen Chem 89(9):1867-1873

Okeke MI, Iroegbu CU, Eze EN, Okoli AS, Esimone CO (2001) Evaluation of extracts of the root of landolphia owerrience for antibacterial activity. J Ethnopharmacol 78:119-127

Patel RV, Kumari P, Rajani DP, Chikhalia KH (2013) Synthesis of coumarin-based 1,3,4-oxadiazol-2ylthio- $N$-phenyl/benzothiazolyl acetamides as antimicrobial and antituberculosis agents. Med Chem Res 22(1):195-210

Patil VS, Nandre KP, Ghosh S, Rao VJ, Chopade BA, Sridhar B, Bhosale SV (2013) Synthesis, crystal structure and antidiabetic activity of substituted (E)-3-(benzo[ $d]$ thiazol-2-ylamino) phenylprop-2-en-1-one. Eur J Med Chem 59:304-309 
Roig-Zamboni V, Cobucci-Ponzano B, Iacono R, Ferrara MC, Germany S, Bourne Y, Sulzenbacher G (2017) Structure of human lysosomal acid $\alpha$-glucosidase-a guide for the treatment of Pompe disease. Nat Commun 8(1):1-10

Sarkar S, Dwivedi J, Chauhan R (2013) Synthesis of 1-[2(substituted phenyl)-4-oxothiazolidin-3-yl]-3-(6-fluro-7-chloro-1,3-benzothiazol-2-yl)-ureas as anthelmintic agent. J Pharm Res 7(5):439-442

Scott KA, Njardarson JT (2018) Analysis of US FDA-approved drugs containing sulfur atoms. Top Curr Chem 376(1):1-34

Sawhney KN, Lemke TL (1983) Chemistry of $\beta$-triketones. 1. Structure of Schiff base intermediates of 2-acyl-1,3-indandiones. J Org Chem 48(23):4326-4329

Shafi S, Alam MM, Mulakayala N, Mulakayala C, Vanaja G, Kalle AM, Alam MS (2012) Synthesis of novel 2-mercaptobenzothiazole and 1,2,3-triazole based bis-heterocycles: their anti-inflammatory and anti-nociceptive activities. Eur J Med Chem 49:324-333

Shareef MA, Sirisha K, Khan I, Sayeed IB, Jadav SS, Ramu G, Babu BN (2019) Design, synthesis, and antimicrobial evaluation of 1,4dihydroindeno[1,2-c]pyrazole tethered carbohydrazide hybrids: exploring their in silico ADMET, ergosterol inhibition and ROS inducing potential. Med Chem Comm 10(5):806-813

Singh SK, Dessalew N, Bharatam PV (2006) 3D-QSAR CoMFA study on indenopyrazole derivatives as cyclin dependent kinase 4 (CDK4) and cyclin dependent kinase 2 (CDK2) inhibitors. Eur J Med Chem 41(11):1310-1319
Trott O, Olson AJ (2010) AutoDock Vina: improving the speed and accuracy of docking with a new scoring function, efficient optimization, and multithreading. J Comput Chem 31(2):455-461

Wagman AS, Boyce RS, Brown SP, Fang E, Goff D, Jansen JM, Nuss JM (2017) Synthesis, binding mode, and antihyperglycemic activity of potent and selective (5-imidazol-2-yl-4-phenylpyrimidin-2-yl)[2-(2-pyridylamino)ethyl] amine inhibitors of glycogen synthase kinase 3. J Med Chem 60(20):8482-8514

Wright JB, Dulin WE, Markillie JH (1964) The antidiabetic activity of 3,5-dimethylpyrazoles. J Med Chem 7(1):102-105

Xiao Z, Storms R, Tsang A (2006) A quantitative starch-iodine method for measuring alpha-amylase and glucoamylase activities. Anal Biochem 351(1):146-148

Yevich JP, New JS, Smith DW, Lobeck WG, Catt JD, Minielli JL, Temple Jr. DL (1986) Synthesis and biological evaluation of 1(1,2-benzisothiazol-3-yl) and (1,2-benzisoxazol-3-yl) piperazine derivatives as potential antipsychotic agents. J Med Chem 29 (3):359-369

Yoshikawa M, Nishida N, Shimoda H, Takada M, Kawahara Y, Matsuda H (2001) Polyphenol constituents from salacia species: quantitative analysis of mangiferin with $\alpha$-glucosidase and aldose reductase inhibitory activities. Yakugaku zasshi J 121(5):371-378

Zhou S, Yang S, Huang G (2017) Design, synthesis and biological activity of pyrazinamide derivatives for anti-Mycobacterium tuberculosis. J Enzym Inhib Med Chem 32(1):1183-1186 